\title{
Cache-Oblivious Range Reporting with Optimal Queries Requires Superlinear Space
}

\author{
Peyman Afshani · Chris Hamilton • Norbert Zeh
}

Received: 3 July 2009 / Revised: 7 April 2010 / Accepted: 30 June 2010 / Published online: 9 April 2011 (C) Springer Science+Business Media, LLC 2011

\begin{abstract}
We consider a number of range reporting problems in two and three dimensions and prove lower bounds on the amount of space used by any cacheoblivious data structure for these problems that achieves the optimal query bound of $\mathrm{O}\left(\log _{B} N+K / B\right)$ block transfers, where $K$ is the size of the query output.

The problems we study are three-sided range reporting, 3-d dominance reporting, and 3-d halfspace range reporting. We prove that, in order to achieve the above query bound or even a bound of $f\left(\log _{B} N, K / B\right)$, for any monotonically increasing function $f(\cdot, \cdot)$, the data structure has to use $\Omega\left(N(\log \log N)^{\varepsilon}\right)$ space. This lower bound holds also for the expected size of any Las-Vegas-type data structure that achieves an expected query bound of at most $f\left(\log _{B} N, K / B\right)$ block transfers. The exponent $\varepsilon$ depends on the function $f(\cdot, \cdot)$ and on the range of permissible block sizes.

Our result has a number of interesting consequences. The first one is a new type of separation between the I/O model and the cache-oblivious model, as deterministic $\mathrm{I} / \mathrm{O}$-efficient data structures with the optimal query bound in the worst case and using linear or $\mathrm{O}\left(N \log ^{*} N\right)$ space are known for the above problems. The second conse-
\end{abstract}

Part of this work was done while P. Afshani had been visiting Dalhousie University.

C. Hamilton was supported by a Killam Predoctoral Scholarship.

N. Zeh was supported in part by the Natural Science and Engineering Research Council of Canada and the Canada Research Chairs programme.

P. Afshani

MADALGO, Department of Computer Science, Aarhus University, IT Parken, Aabogade 34, 8200

Aarhus N, Denmark

e-mail: peyman@madalgo.au.dk

C. Hamilton · N. Zeh $(\bowtie)$

Faculty of Computer Science, Dalhousie University, Halifax, NS, B3H 1W5, Canada

e-mail: nzeh@cs.dal.ca

C. Hamilton

e-mail: chamilton@cs.dal.ca 
quence is the non-existence of linear-space cache-oblivious persistent B-trees with optimal 1-d range reporting queries.

Keywords Cache-obliviousness $\cdot$ Lower bound $\cdot$ Range reporting

\section{Introduction}

Range reporting is a well studied fundamental problem in computational geometry. Given a set $S$ of points in $\mathbb{R}^{d}$, the goal is to preprocess $S$ so that, for any query range $q$ of a given shape, all points in $S \cap q$ can be reported efficiently. Typical query shapes include axis-aligned boxes, circles, simplices, and halfspaces. To indicate the type of permissible queries, the problem is then referred to more specifically as orthogonal, circular, simplex or halfspace range reporting. Three-sided range reporting is a special case of 2-d orthogonal range reporting that considers axis-aligned boxes whose top boundaries are fixed at $y=+\infty$. Dominance reporting is another important special case of orthogonal range reporting: given a query point $q$, the problem is to report all points in $S$ that are dominated by $q$, that is, whose coordinates are less than $q$ 's in all dimensions. These different query types are illustrated in Fig. 1.

Most previous work on this type of problem has focused on standard models of computation, such as the RAM model or the pointer machine model. The distinguishing feature of these models is that the access cost to a data item is independent of the location where the item is stored in memory. These models are useful for studying the fundamental computational difficulty of a problem, but they ignore that in reality the time to access an item can vary by a factor of up to $10^{6}$ depending on its present location (disk, internal memory, CPU cache, etc.).

A number of models have been proposed to capture the non-uniform access costs in real memory hierarchies. See [31] for a survey. The two most widely adopted ones are the input/output model (or I/O model) [6] and the cache-oblivious model [18]. Their success is due to the balance they provide between simplicity, in order to allow the design and analysis of sophisticated algorithms, and accuracy in predicting the performance of algorithms on real memory hierarchies.

The I/O model considers two levels of memory: a fast internal memory with the capacity to hold $M$ data items, and a slow but conceptually unlimited external memory. All computation has to happen on data in internal memory. The transfer of data between internal and external memory happens in blocks of $B$ consecutive data items; the complexity of an algorithm is the number of such block transfers it performs.

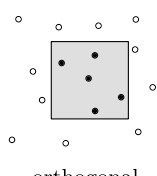

orthogonal

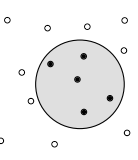

circular

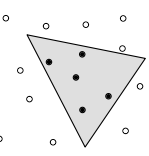

simplex

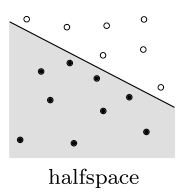

halfspace
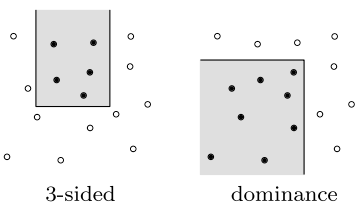

Fig. 1 Standard query types in 2-d 
The cache-oblivious model provides a simple framework for designing algorithms for multi-level memory hierarchies, while using the simple two-level I/O model for the analysis. In this model, the algorithm is oblivious of the memory hierarchy and, thus, cannot initiate block transfers explicitly. Instead, the swapping of data between internal and external memory is the responsibility of a paging algorithm, which is assumed to be offline optimal, that is, to perform the minimum number of block transfers possible for the memory access sequence of the algorithm. Since the memory parameters are used only in the analysis, the analysis applies to any two consecutive levels of the memory hierarchy. In particular, if the analysis shows that the algorithm is optimal with respect to two levels of memory, it is simultaneously optimal at all levels of the memory hierarchy. See [18] for a more detailed discussion of this model and for a justification of the optimality assumption of the paging algorithm.

In this paper, we study three-sided range reporting, 3-d dominance reporting, and 3 -d halfspace range reporting in the cache-oblivious model. We prove that any cacheoblivious data structure for these problems that achieves the optimal (or even a much weaker) query bound has to use asymptotically more space than a data structure with the same query bound in the I/O model.

\subsection{Related Work}

In the I/O model, much work has focused on orthogonal range reporting. A number of linear-space data structures have been proposed that achieve a query bound of $\mathrm{O}(\sqrt{N / B}+K / B)$ block transfers in two dimensions and $\mathrm{O}\left((N / B)^{1-1 / d}+K / B\right)$ block transfers in higher dimensions $[9,19,20,22,26,28]$, where $K$ is the number of reported points. The same bounds have been obtained in the cache-oblivious model [5, 11]. In 2-d, Arge et al. [8] showed that $\Theta\left(N \log N / \log \log _{B} N\right)$ space is sufficient and necessary to obtain a query bound of $\mathrm{O}\left(\log _{B} N+K / B\right)$ block transfers for orthogonal range reporting in the $\mathrm{I} / \mathrm{O}$ model. The main tool used to prove the upper bound is an I/O-efficient version [8] of McCreight's priority search tree [25] with a query bound of $\mathrm{O}\left(\log _{B} N+K / B\right)$ block transfers for three-sided range reporting queries and using linear space. The lower bound is obtained in the indexability model of [21] (also see Sect. 2). Note that the lower bound shows that any data structure achieving the optimal query bound for $B=N^{\varepsilon}$ must use $\Omega(N \log N)$ space. In particular, any cache-oblivious data structure supporting 2-d orthogonal range reporting queries in the optimal query bound has to use $\Omega(N \log N)$ space.

In the cache-oblivious model, three data structures have been proposed that achieve a query bound of $\mathrm{O}\left(\log _{B} N+K / B\right)$ block transfers for three-sided range reporting, but using $\mathrm{O}(N \log N)$ space. The first one, by Agarwal et al. [5], works only if $\log \log B$ is an integer. The second and third data structures, by Arge et al. [10] and Arge and Zeh [7], remove this restriction. The data structure by Arge and Zeh is obtained using standard techniques from a linear-space data structure for optimal 2-d dominance reporting queries presented in the same paper. By applying the same techniques to the $\mathrm{O}(N \log N)$-space three-sided range reporting data structures of $[5,7,10]$, it is easy to obtain cache-oblivious 2 -d orthogonal range reporting data structures that use $\mathrm{O}\left(N \log ^{2} N\right)$ space and achieve the optimal query bound of $\mathrm{O}\left(\log _{B} N+K / B\right)$ block transfers. 
Table 1 A summary of known upper bounds for three-sided range reporting, 3-d dominance reporting and 3-d halfspace range reporting. For the sake of clarity, O-notation has been omitted

\begin{tabular}{lllll}
\hline Query type & Model & Space & Query bound & References \\
\hline 2-d three-sided & internal memory & $N$ & $\log N+K$ & {$[25]$} \\
& I/O model & $N$ & $\log _{B} N+K / B$ & {$[8]$} \\
& cache-oblivious model & $N \log N$ & $\log _{B} N+K / B$ & {$[3,5,7,10]$} \\
3-d dominance & internal memory & $N$ & $\log N+K$ & {$[1,24]$} \\
& I/O model & $N$ & $\log _{B} N+K / B$ & {$[1]$} \\
& cache-oblivious model & $N \log N$ & $\log _{B} N+K / B$ & {$[3]$} \\
3-d halfspace & internal memory & $N$ & $\log N+K$ & {$[2]$} \\
& I/O model & $N \log *$ & $\log _{B} N+K / B$ & {$[2]$} \\
& cache-oblivious model & $N \log N$ & $\log _{B} N+K / B$ & {$[3]$} \\
\hline
\end{tabular}

For 3-d dominance reporting, Vengroff and Vitter [30] presented a data structure with a query bound of $\mathrm{O}\left(\left(\log \log \log _{B} N\right) \log (N / B)+K / B\right)$ block transfers and using $\mathrm{O}(N \log (N / B))$ space in the $\mathrm{I} / \mathrm{O}$ model. The query bound can be reduced to $\mathrm{O}\left(\log _{B} N+K / B\right)$ block transfers by choosing the parameters in the data structure more carefully [31]. Afshani [1] showed that an optimal query bound can in fact be obtained using linear space, raising the question whether this result can be achieved also in the cache-oblivious model. In [3], we show that the optimal query bound can indeed be achieved by a cache-oblivious data structure, but our data structure uses $\mathrm{O}(N \log N)$ space.

Halfspace range reporting in 3-d has a longer history, in part because it can be used to solve other problems, such as 2-d circular range reporting and 2-d $K$-nearest neighbour searching. In internal memory, Chan described an $\mathrm{O}(N \log N)$-space data structure with an expected query time of $\mathrm{O}(\log N+K)$ [16]. Building on these ideas, Agarwal et al. [4] obtained an $\mathrm{O}(N \log N)$-space data structure with an expected query bound of $\mathrm{O}\left(\log _{B} N+K / B\right)$ block transfers in the I/O model. Further research led to the development of internal-memory data structures with the optimal query bound in the worst case and using $\mathrm{O}(N \log \log N)$ space [17, 27]. The same improvements can be carried over to the I/O model. Recently, Afshani and Chan [2] described a linear-space data structure with the optimal query bound in internal memory and an $\mathrm{O}\left(N \log ^{*} N\right)$-space data structure that answers queries using $\mathrm{O}\left(\log _{B} N+K / B\right)$ block transfers in the I/O model. In [3], we show how to achieve the optimal query bound in the cache-oblivious model, but using $\mathrm{O}(N \log N)$ space. Table 1 summarizes these results.

\subsection{New Results}

As discussed in the previous section, there exist linear- or $\mathrm{O}\left(N \log ^{*} N\right)$-space data structures that achieve the optimal query bound of $\mathrm{O}\left(\log _{B} N+K / B\right)$ block transfers for three-sided range reporting, 3-d dominance reporting, and 3-d halfspace range reporting in the I/O model. In contrast, the best known data structures achieving the 
same query bound in the cache-oblivious model use $\mathrm{O}(N \log N)$ space. This raises the question whether linear-space cache-oblivious data structures with the optimal query bound exist for these problems. In this paper, we give a negative answer to this question. We prove that any cache-oblivious data structure for three-sided range reporting, 3-d dominance reporting or 3-d halfspace range reporting that achieves a query bound of $f\left(\log _{B} N, K / B\right)$, for any monotonically increasing function $f(\cdot, \cdot)$, has to use $\Omega\left(N(\log \log N)^{\varepsilon}\right)$ space. ${ }^{1}$ This lower bound holds also for the expected size of Las-Vegas-type data structures, that is, data structures with randomized construction and query algorithms that guarantee correct query answers but achieve the desired query bound only in the expected case. The exponent $\varepsilon$ depends on the function $f(\cdot, \cdot)$ and on the range of permissible block sizes. Our results are shown in a multi-level extension of the indexability model of [21], which is discussed in more detail in Sect. 2. In particular, they are independent of the set of supported internalmemory operations (comparisons, algebraic operations, etc.).

As a consequence of our lower bound for three-sided range reporting, it follows that there is no linear-space cache-oblivious persistent $B$-tree that achieves the optimal 1-d range reporting bound of $\mathrm{O}\left(\log _{B} N+K / B\right)$ block transfers in the worst or expected case, while such a data structure exists in the I/O model [13].

There have been previous results showing that the cache-oblivious model is less powerful than the I/O model. Brodal and Fagerberg [15] established a lower bound on the amount of main memory (as a function of $B$ ) necessary for optimal cacheoblivious sorting, while Bender et al. [14] proved that cache-oblivious searching has to cost a constant factor more than the search bound achieved in the I/O model using B-trees [12]. Our result is the first to establish an asymptotically growing gap between the space used by cache-oblivious and I/O-efficient data structures.

The key to obtaining our result is the construction of a hard point set and of a set of hard queries over this point set in combination with techniques to explicitly use the multi-level structure of the cache-oblivious model. Previous lower bound proofs for range reporting problems in the $\mathrm{I} / \mathrm{O}$ model $[8,21,23,29]$ involved the construction of a hard point set together with a set of many "sufficiently different" queries of the same size. Combined with counting arguments, this ensured that the point set cannot be represented in linear space while guaranteeing a certain proximity (on disk) of the points reported by each query. The problems we study in this paper allow linear-space or $\mathrm{O}\left(N \log ^{*} N\right)$-space solutions in the $\mathrm{I} / \mathrm{O}$ model $[1,2,8]$, as well as linear-space cache-oblivious solutions for queries of any fixed output size $[3,5,10]$. This means the previous techniques are ineffective for our purposes. In order to force a given point set to be hard for the problems we study, we construct many queries of different sizes. Combined with the multi-level nature of the cache-oblivious model, this allows us to create many incompatible proximity requirements for subsets of the point set and, thus, force duplication. It should be noted here that our construction of a hard point set (as well as the proof of Lemma 4) is inspired by a similar construction used by Afshani and Chan to prove a lower bound on the shallow partition theorem [2].

\footnotetext{
${ }^{1}$ We call a function $f(\cdot, \cdot)$ monotonically increasing if $x \geq x^{\prime}$ and $y \geq y^{\prime}$ imply $f(x, y) \geq f\left(x^{\prime}, y^{\prime}\right)$.
} 


\section{A Multi-Level Indexability Model}

The results in this paper are proved in a multi-level extension of the indexability model of [21]. In particular, apart from a change of notation, the model of [21] is the same as our model restricted to one block size. This model allows us to prove lower bounds for (cache-oblivious and cache-aware) data structures for multi-level memory hierarchies without any restrictions of the set of supported internal-memory operations. The two main concepts of the model are those of workload, which captures a particular combination of problem instance and memory hierarchy, and indexing scheme, which provides an abstraction of a data structure. Next we define these two concepts, as well as the size and efficiency of an indexing scheme, and discuss their relationships to the data structure concepts they capture.

A workload is a triple $\mathcal{W}=(S, Q, \mathcal{B})$, where $S$ is a set, $Q$ is a set of subsets of $S$, and $\mathcal{B}$ is a set of integers. We call $S$ the base set, $Q$ the query set, and the elements of $\mathcal{B}$ block sizes. Intuitively, $S$ is the set of elements to be stored in a data structure, every element of $Q$ contains the set of elements in $S$ to be reported by a particular query, and the elements of $\mathcal{B}$ are the cache block sizes of a particular memory hierarchy. We call a workload realizable as a range reporting problem of a given type if the elements in $S$ can be identified with points in $\mathbb{R}^{d}$ so that for every set $q \in Q$ there exists a query range of the given type containing exactly those points in $S$ that correspond to the elements of $q$.

A block cover of $S$ with block size $B$ (short: $B$-cover) is a set of subsets (blocks) of $S$, each of size at most $B$ and such that their union is $S$. An indexing scheme for the workload $\mathcal{W}=(S, Q, \mathcal{B})$ is a set of block covers of $S$, one for each block size in $\mathcal{B}$. Intuitively, an indexing scheme provides an abstraction of a data structure, as every layout of a concrete data structure $\mathcal{D}$ in external memory can place only $B_{i}$ elements of $S$ into each cache block of size $B_{i} \in \mathcal{B}$. We refer to the indexing scheme representing this placement of elements into cache blocks as $\mathcal{I}_{\mathcal{D}}$.

For a workload $\mathcal{W}=(S, Q, \mathcal{B})$ and an indexing scheme $\mathcal{I}$ for $\mathcal{W}$, we say that a $B_{i}$-cover $\mathcal{C}_{i}$ in $\mathcal{I}$ is $f$-efficient, for a function $f(\cdot, \cdot)$, if every query $q \in Q$ can be covered with at most $f\left(\log _{B_{i}} N, K / B_{i}\right)$ blocks in $\mathcal{C}_{i}$, where $N:=|S|$ and $K:=|q|$. The indexing scheme $\mathcal{I}$ is $f$-efficient if all its block covers are $f$-efficient. Applied to the indexing scheme $\mathcal{I}_{\mathcal{D}}$ corresponding to a data structure $\mathcal{D}$, this captures the number of cache blocks at each level of the memory hierarchy that need to be retrieved from $\mathcal{D}$ to answer any query $q \in Q$. As in [21], the cost of locating these blocks is ignored. In particular, a lower bound proved in this model is independent of the set of supported internal-memory operations.

For a block cover $\mathcal{C}$ of $S$, the multiplicity $\mu_{\mathcal{C}}(x)$ of an element $x \in S$ is the number of blocks in $\mathcal{C}$ that contain $x$. For an indexing scheme $\mathcal{I}$ for a workload $\mathcal{W}=(S, Q, \mathcal{B})$, the multiplicity of $x$ is defined as $\mu_{\mathcal{I}}(x)=\max _{\mathcal{C}_{i}} \mu_{\mathcal{C}_{i}}(x)$, where $\mathcal{C}_{i}$ ranges over all block covers in $\mathcal{I}$. The size of $\mathcal{I}$ is $\sum_{x \in S} \mu_{\mathcal{I}}(x)$. For the indexing scheme $\mathcal{I}_{\mathcal{D}}$ representing a data structure $\mathcal{D}$ and a $B_{i}$-cover $\mathcal{C}_{i}$ in $\mathcal{I}_{\mathcal{D}}$, every element $x \in S$ is stored in $\mu_{\mathcal{C}_{i}}(x)$ cache blocks of size $B_{i}$ in $\mathcal{D}$. Hence, the multiplicity $\mu_{\mathcal{C}_{i}}(x)$ of an element $x \in S$ is a lower bound on the number of times $x$ is stored in $\mathcal{D}$. Since this is true for every block cover in $\mathcal{I}_{\mathcal{D}}, \mu_{\mathcal{I}_{\mathcal{D}}}(x)$ is also a lower bound on the number of times $x$ is stored in $\mathcal{D}$, and the size of $\mathcal{I}_{\mathcal{D}}$ is a lower bound on the size of $\mathcal{D}$. Thus, 
given a workload $\mathcal{W}=(S, Q, \mathcal{B})$, it suffices to prove a lower bound on the size of any $f$-efficient indexing scheme for $\mathcal{W}$ in order to obtain a lower bound on the size of any data structure that achieves a query cost of $f\left(\log _{B} N, K / B\right)$ block transfers for all queries in $Q$ and all block sizes $B \in \mathcal{B}$.

The workloads $\mathcal{W}=(S, Q, \mathcal{B})$ we construct in this paper have a large number of block sizes in $\mathcal{B}$. Thus, our lower bounds apply to cache-aware data structures with query cost bounded by $f\left(\log _{B} N, K / B\right)$ block transfers only under the assumption of a very deep memory hierarchy. A cache-oblivious data structure, on the other hand, is independent of the specific memory hierarchy. If it achieves a query bound of $f\left(\log _{B} N, K / B\right)$ block transfers for an arbitrary two-level hierarchy with block size $B$, it achieves this query bound at all levels of a multi-level hierarchy with the block sizes in $\mathcal{B}$. Thus, any lower bound we prove on the size of an $f$-efficient indexing scheme for $\mathcal{W}$ applies to any cache-oblivious data structure with a query cost of $f\left(\log _{B} N, K / B\right)$ block transfers.

\section{A Lower Bound for Three-Sided Range Reporting}

In this section, we present the main result of our paper: a lower bound on the space used by any deterministic cache-oblivious data structure that supports three-sided range reporting queries using at most $f\left(\log _{B} N, K / B\right)$ block transfers in the worst case, as stated in Theorem 1 . The lower bounds for 3 -d dominance reporting and 3-d halfspace range reporting are obtained from this result using reductions and are discussed in Sect. 5. The same section discusses how to extend the lower bound to Las-Vegas-type randomized data structures.

Theorem 1 Let $f(\cdot, \cdot)$ be a monotonically increasing function, and $0<\delta \leq 1 / 2$ a constant. Any cache-oblivious data structure capable of answering three-sided range reporting queries using at most $f\left(\log _{B} N, K / B\right)$ block transfers in the worst case, for every block size $B \leq N^{2 \delta}$, must use $\Omega\left(N(\log \log N)^{\varepsilon}\right)$ space, where $\varepsilon:=$ $1 / f\left(\delta^{-1}, 1\right)$.

We prove Theorem 1 by constructing a workload $\mathcal{W}$ realizable as a three-sided range reporting problem such that any $f$-efficient indexing scheme for $\mathcal{W}$ has size $\Omega\left(N(\log \log N)^{\varepsilon}\right)$; see Lemma 1 below. As discussed in Sect. 2, this implies Theorem 1 . However, while an indexing scheme and its efficiency are defined without reference to cache sizes, a data structure can take advantage of the amount of available cache to speed up its queries. Our proof of Lemma 1 considers block sizes between $N^{\delta}$ and $N^{2 \delta}$. Thus, a sufficiently strong tall cache assumption $\left(M=\omega\left(B^{1 / \delta}\right)\right.$, for all $0<\delta \leq 1 / 2$ ) implies that the entire point set fits in cache, and the queries in $Q$ can be answered without any block transfers after loading $S$ into cache. This means that Theorem 1 holds only under the assumption that $M$ is polynomial in $B$. In practice, this is a reasonable assumption. In the remainder of this section, we prove the following lemma.

Lemma 1 Let $f(\cdot, \cdot)$ be a monotonically increasing function, and $0<\delta \leq 1 / 2$ a constant. For every integer $N>0$, there exists a workload $\mathcal{W}=(S, Q, \mathcal{B})$ realizable 
as a three-sided range reporting problem, with $|S|=N$ and $B_{i} \leq N^{2 \delta}$, for all $B_{i} \in \mathcal{B}$, and such that any $f$-efficient indexing scheme for $\mathcal{W}$ has size $\Omega\left(N(\log \log N)^{\varepsilon}\right)$, where $\varepsilon:=1 / f\left(\delta^{-1}, 1\right)$.

First we show that, to prove Lemma 1 , it suffices to focus on the case $\delta=1 / 2$, that is, to allow arbitrarily large block sizes $B_{i} \leq N$ in $\mathcal{B}$.

Lemma 2 If Lemma 1 holds for $\delta=1 / 2$, it holds for any $0<\delta \leq 1 / 2$.

Proof Consider a particular choice of $N, f$, and $\delta$ in Lemma 1, and let $N^{\prime}:=N^{2 \delta}$ and $f^{\prime}(x, y):=f(x /(2 \delta), y)$. Since we assume that Lemma 1 holds for $\delta=1 / 2$, there exist a workload $\mathcal{W}^{\prime}=\left(S^{\prime}, Q^{\prime}, \mathcal{B}\right)$ realizable as a three-sided range reporting problem, with $\left|S^{\prime}\right|=N^{\prime}$ and $B_{i} \leq N^{\prime}$, for all $B_{i} \in \mathcal{B}$, and such that any $f^{\prime}$-efficient indexing scheme for $\mathcal{W}^{\prime}$ has size $\Omega\left(N^{\prime}\left(\log \log N^{\prime}\right)^{\varepsilon}\right)$, where $\varepsilon:=1 / f^{\prime}(2,1)=1 / f\left(\delta^{-1}, 1\right)$.

Now we construct a workload $\mathcal{W}=(S, Q, \mathcal{B})$ with $|S|=N$ as follows. Let $m:=N / N^{\prime}$. For $1 \leq i \leq m$, we define sets $S_{i}:=\left\{(x, i) \mid x \in S^{\prime}\right\}$ and $Q_{i}:=\{\{(x, i) \mid$ $\left.x \in q\} \mid q \in Q^{\prime}\right\}$. The sets $S$ and $Q$ are defined as $S:=S_{1} \cup S_{2} \cup \cdots \cup S_{m}$ and $Q:=Q_{1} \cup Q_{2} \cup \cdots \cup Q_{m}$. Since $\mathcal{W}^{\prime}$ is realizable as a three-sided range reporting problem, so is $\mathcal{W}$ : it suffices to place $m$ copies of the point set representing $S^{\prime}$ side by side.

The workload $\mathcal{W}_{i}=\left(S_{i}, Q_{i}, \mathcal{B}\right)$ is the same as $\mathcal{W}^{\prime}$ after renaming every element $x \in S^{\prime}$ to $(x, i)$. Thus, every $f^{\prime}$-efficient indexing scheme for $\mathcal{W}_{i}$ has size $\Omega\left(N^{\prime}\left(\log \log N^{\prime}\right)^{\varepsilon}\right)$. Now observe that $f^{\prime}\left(\log _{B} N^{\prime}, K / B\right)=f\left(\left(\log _{B}\left(N^{2 \delta}\right)\right) /(2 \delta)\right.$, $K / B)=f\left(\log _{B} N, K / B\right)$, that is, the restriction of every $f$-efficient indexing scheme for $\mathcal{W}$ to the elements of $S_{i}$ is an $f^{\prime}$-efficient indexing scheme for $\mathcal{W}_{i}$. This implies that every $f$-efficient indexing scheme for $\mathcal{W}$ has size $\Omega\left(m N^{\prime}\left(\log \log N^{\prime}\right)^{\varepsilon}\right)$ $=\Omega\left(N(\log \log N)^{\varepsilon}\right)$.

By Lemma 2, it suffices to prove Lemma 1 for arbitrarily large block sizes. For a given size $N$ of the set $S$, we construct the sets $S, Q$, and $\mathcal{B}$ in the workload $\mathcal{W}=$ $(S, Q, \mathcal{B})$ recursively. Since the workload is to be realizable as a three-sided range reporting problem, we do not distinguish between the elements in $S$ and the points in $\mathbb{R}^{2}$ representing them, nor between the queries in $Q$ and their corresponding threesided query ranges.

The recursive construction of the point set $S$ is illustrated in Fig. 2a. At the first level of recursion, we divide the plane into a $t \times 2^{t-1}$-grid $T$, for a parameter $t$ to be chosen later, and place different numbers of points into its cells. The points within each cell are arranged by dividing the cell into a $t \times 2^{t-1}$-grid of subcells and distributing the points over those subcells. This process continues recursively as long as each cell of the current subgrid contains more than $\sqrt{N}$ points.

The construction of the query set $Q$ follows the recursive construction of the point set $S$. Each query at the top level comprises a union of cells of $T$ chosen so that each top-level query outputs roughly the same number of points. For each grid cell, we construct a set of queries over the subgrid in this cell in a similar fashion. We repeat this construction recursively until we reach the last level in the recursive construction of the point set $S$. 


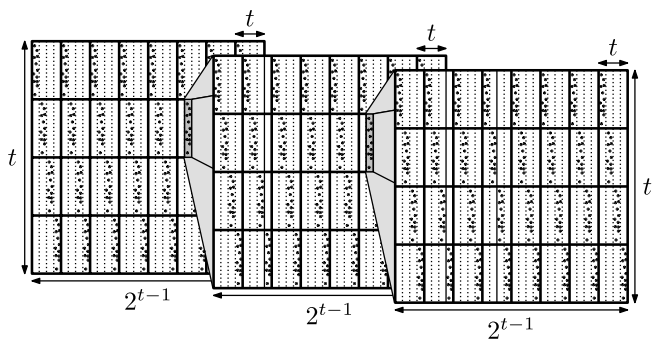

(a)

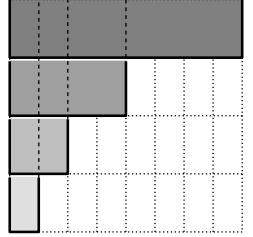

(b)

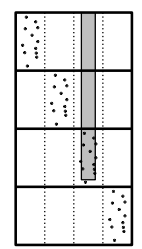

(c)

Fig. 2 (a) The recursive construction of the point set. Fat solid lines bound grid cells, dotted lines separate subcolumns. (b) The set of queries in $Q_{T}$. Only one query is shown for each level of $Q_{T}$. (c) Queries at recursive levels output only points from their subgrids

The main idea now is to prove that, by choosing the top-level queries in $Q$ appropriately and including an appropriate block size in $\mathcal{B}$, we can ensure that, for every $f$-efficient indexing scheme $\mathcal{I}$ for $\mathcal{W}$, there exists a grid cell in $T$ at least half of whose points have multiplicity $\Omega\left(t^{\varepsilon}\right)$ in $\mathcal{I}$. For each subgrid at the next level of recursion, we add another block size to $\mathcal{B}$ that ensures that this subgrid has a cell at least half of whose points have multiplicity $\Omega\left(t^{\varepsilon}\right)$, and so on for every level of recursion. By making the recursion sufficiently deep, we achieve that at least a constant fraction of the points have multiplicity $\Omega\left(t^{\varepsilon}\right)$. The required recursion depth depends on $t$, and the largest value of $t$ that allows for a sufficient recursion depth is $t \approx \log \log N$ (see remark at the end of Sect. 3.1). This gives the lower bound stated in Lemma 1.

We divide the details of our proof into two parts. In Sect. 3.1, we discuss the construction of the point set $S$ and of the query set $Q$ more precisely and prove that Lemma 1 follows if we can force at least half the points in one cell of each of the subgrids in the recursive construction to have the claimed multiplicity. In Sect. 3.2, we discuss how to achieve this duplication of points for each subgrid by including appropriate block sizes in $\mathcal{B}$.

\subsection{The Point Set and Query Set}

To define the point set $S$, we construct a $t \times 2^{t-1}$-grid $T$, for a parameter $t:=$ $(\log \log N) / 4$. This parameter remains fixed throughout the recursive construction. We refer to the grid cell in row $i$ and column $j$ as $T_{i j}$, for $1 \leq i \leq t$ and $1 \leq j \leq 2^{t-1}$. Every column of $T$ is divided into $t$ subcolumns, which also splits each cell $T_{i j}$ into $t$ subcells $T_{i j k}$, for $1 \leq k \leq t$. We now place $2^{i-1} N_{1}$ points into each cell in row $i$, where $N_{1}:=N /\left(2^{2 t-1}-2^{t-1}\right)$. The points in cell $T_{i j}$ are placed into subcell $T_{i j i}$. This is illustrated in Fig. 2a. Observe that this ensures that each column of the grid receives $\left(2^{t}-1\right) N_{1}$ points. Since there are $2^{t-1}$ columns, the total number of points in the grid is $\left(2^{2 t-1}-2^{t-1}\right) N_{1}=N$. The layout of the points within each cell is now obtained by applying the same procedure recursively to the set of points assigned to each cell. The recursion stops when the smallest cell in the current subgrid receives at most $\sqrt{N}$ points.

The query set $Q$ is constructed by following the recursive construction of $S$. For the top-level grid $T$, we construct a set $Q_{T}$ of queries consisting of $t$ levels. For 
$1 \leq i \leq t$, level $i$ contains $2^{i-1}$ queries, the $k$ th of which is the union of all grid cells $T_{i^{\prime} j^{\prime}}$ satisfying $0<i^{\prime} \leq i$ and $(k-1) 2^{t-i}<j^{\prime} \leq k 2^{t-i}$; see Fig. 2 b. It is easily verified that every query in $Q_{T}$ contains between $2^{t-1} N_{1}$ and $\left(2^{t}-1\right) N_{1}$ points. Note that, even though we specify these queries as unions of grid cells, that is, effectively, as four-sided queries, we can move their top boundaries to infinity without changing the set of points they report. To complete the construction of the query set $Q$, we apply the same construction recursively to each cell, adding a query set $Q_{T^{\prime}}$ to $Q$, for each subgrid $T^{\prime}$ in the recursive construction of $S$. Again, we can move the top boundary of each query in $Q_{T^{\prime}}$ to infinity to make it three-sided without changing the set of points it reports. Indeed, this clearly does not change the set of points from $T^{\prime}$ reported by the query, and the staggered layout of the points in each grid column into $x$-disjoint subcolumns ensures that there are no points in $S$ that belong to the $x$-range of $T^{\prime}$ but are outside its $y$-range. This is illustrated in Fig. 2c.

Now let $\mathcal{W}=(S, Q, \mathcal{B})$ be a workload with $S$ and $Q$ as just defined. The following lemma provides the framework we use to prove Lemma 1.

Lemma 3 Let $\mathcal{I}$ be an arbitrary indexing scheme for $\mathcal{W}$ and assume that every subgrid $T^{\prime}$ in the construction of $S$ has a cell at least half of whose points have multiplicity $\Omega(m)$ in $\mathcal{I}$. Then the size of $\mathcal{I}$ is $\Omega(m N)$.

Proof We construct a set of disjoint cells such that at least half of the points in each cell have multiplicity $\Omega(m)$ and the total number of points in these cells is $\Omega(N)$. This proves that $\mathcal{I}$ has size $\Omega(m N)$.

To construct this set of cells, we apply the following recursive selection process, starting with $T^{\prime}=T$. By the assumption of the lemma, the grid $T^{\prime}$ has a cell $C^{\prime}$ at least half of whose points have multiplicity $\Omega(m)$. We add $C^{\prime}$ to the set of selected cells and recurse on the subgrids in each of the remaining cells of $T^{\prime}$ unless the current grid $T^{\prime}$ is already at the lowest level of recursion in the construction of the point set $S$.

The set of cells selected in this fashion is easily seen to be disjoint, since we recursively select cells only from subgrids in cells not selected at the current level. We call a point selected if it is contained in one of the selected cells. We have to show that there are $\Omega(N)$ selected points in $T$.

For a grid $T^{\prime}$ containing $N^{\prime}$ points, the construction of $S$ implies that each cell contains at least $N^{\prime} /\left(2^{2 t-1}-2^{t-1}\right)>N^{\prime} / 4^{t}$ points. This allows us to show that at most $N^{\prime}\left(1-4^{-t}\right)^{r^{\prime}+1}$ points from $T^{\prime}$ are not selected by the recursive selection process, where $r^{\prime}$ denotes the minimum recursion depth inside the cells of $T^{\prime}$. Indeed, if $r^{\prime}=0$, exactly the points in $C^{\prime}$ are selected, leaving at most $N^{\prime \prime}=N^{\prime}\left(1-4^{-t}\right)$ points in $T^{\prime}$ unselected. If $r^{\prime}>0$, we observe that, by induction, at most $N^{\prime \prime}\left(1-4^{-t}\right)^{r^{\prime}}=$ $N^{\prime}\left(1-4^{-t}\right)^{r^{\prime}+1}$ points in the cells of $T^{\prime}$ other than $C^{\prime}$ are not selected, while all points in $C^{\prime}$ are selected.

Since the recursion stops when the smallest cell of the current subgrid contains at most $\sqrt{N}$ points and, as just argued, every cell of a grid $T^{\prime}$ containing $N^{\prime}$ points contains at least $N^{\prime} / 4^{t}$ points, the recursion depth $r$ inside each cell of the top-level 
grid $T$ is at least

$$
\log _{4^{t}} \frac{N}{\sqrt{N}}=\frac{(\log N) / 2}{2 t}=\frac{\log N}{\log \log N}>\sqrt{\log N},
$$

for $N$ sufficiently large. Hence, the number of points in $T$ that are not selected is at most

$$
N\left(1-4^{-t}\right)^{r+1} \leq N e^{-(r+1) / 4^{t}} \leq N / e
$$

because $t=(\log \log N) / 4$. Therefore, at least $(1-1 / e) N=\Omega(N)$ points in $T$ are selected. This completes the proof.

The proof of Lemma 3 is the reason we cannot choose a value of $t=\omega(\log \log N)$. To bound the number of unselected points by $N / e$, we need that $r+1 \geq 4^{t}$. Since the recursion depth $r$ is at most logarithmic in $N$, we cannot have $t=\omega(\log \log N)$.

\subsection{Forcing Duplication in at Least One Cell}

In this section, we prove that there exists a block size $B_{T^{\prime}}$, for every subgrid $T^{\prime}$ in the recursive construction of $S$, such that every $f$-efficient $B_{T^{\prime}}$-covering $\mathcal{C}$ of $S$ has the property that there exists a cell of $T^{\prime}$ at least half of whose points have multiplicity $\Omega\left(t^{\varepsilon}\right)$ in $\mathcal{C}$. By including the block size $B_{T^{\prime}}$ in $\mathcal{B}$, for every subgrid $T^{\prime}$ in the recursive construction of $S$, we obtain that every $f$-efficient indexing scheme $\mathcal{I}$ for the workload $\mathcal{W}=(S, Q, \mathcal{B})$ satisfies Lemma 3 with $m=t^{\varepsilon}$. Lemma 1 follows.

The argument is the same for all subgrids $T^{\prime}$. Therefore, we focus on the top-level grid $T$ and its corresponding query set $Q_{T}$ in the remainder of this section. Recall that every query in $Q_{T}$ contains between $N_{1} 2^{t-1}$ and $N_{1}\left(2^{t}-1\right)$ points. We choose a block size $B_{T}:=\left(2^{t}-1\right) N_{1}$. Then every query $q \in Q_{T}$ satisfies $|q| \leq B_{T}$. Furthermore, $B_{T} \geq N_{1} \geq \sqrt{N}$ and, therefore, $\log _{B_{T}} N \leq 2$. Thus, by the monotonicity of $f(\cdot, \cdot)$, every $f$-efficient $B_{T}$-covering $\mathcal{C}$ of $S$ covers every query in $Q_{T}$ with at most $\alpha:=f(2,1)$ blocks.

In the remainder of this section, we fix an arbitrary $f$-efficient $B_{T}$-covering $\mathcal{C}$ of $S$. We can represent every block in $\mathcal{C}$ by a unique colour and assign corresponding colour sets to the points in $S$ and queries in $Q_{T}$. The colour set $C(p)$ of a point $p$ comprises the colours of those blocks in $\mathcal{C}$ that contain $p$. Every query $q \in Q_{T}$ can be covered using at most $\alpha$ blocks in $\mathcal{C}$; we fix such a set of $\alpha$ blocks and define $C(q)$ to be the set of their colours. Our goal can now be rephrased as showing that there exists a cell in $T$ at least half of whose points have $\Omega\left(t^{\varepsilon}\right)$ colours, for $\varepsilon:=1 / \alpha$. We do this in two steps. In Lemma 4, we show that there exists a set of $s:=\Omega(t / \log \alpha)$ cells in the same column of $T$ such that half of the points in each cell are "exposed" in a sense defined below. Then we show that there exists at least one cell among these $s$ cells such that every exposed point in this cell has at least $s^{\varepsilon}=\Omega\left(t^{\varepsilon}\right)$ colours. $^{2}$

Consider a subset $Z$ of cells of $T$. Each cell $c \in Z$ has a corresponding query $q_{c} \in Q_{T}$ that contains the points in $c$ but not the points in the cell of $T$ immediately

\footnotetext{
${ }^{2}$ Since $\alpha=1 / \varepsilon$, we have $s^{\varepsilon}=\Omega\left((t / \log (1 / \varepsilon))^{\varepsilon}\right)$, and it is not hard to show that $(\log (1 / \varepsilon))^{-\varepsilon}>1 / 2$, for all $0<\varepsilon<1$.
} 
below $c$. Let $Q_{Z}$ be the set of queries corresponding to the cells in $Z$. We say a query $q$ covers a point $p$ if $C(p) \cap C(q) \neq \emptyset$, that is, $p$ is contained in one of the $\alpha$ blocks of $\mathcal{C}$ chosen to cover $q$. A point $p$ in the $i$ th row of $T$ is $Z$-exposed if no query $q_{c} \in Q_{Z}$ that belongs to a level $j<i$ in $Q_{T}$ covers $p$. A cell of $T$ is $Z$-exposed if at least half its points are $Z$-exposed. An observation we use in the proof of the following lemma is that a $Z$-exposed point or cell is also $Z^{\prime}$-exposed, for every subset $Z^{\prime} \subseteq Z$.

Lemma 4 There exists a column in $T$ containing a set $Z$ of $\Omega(t / \log \alpha) Z$-exposed cells.

Proof Let $h$ be a parameter to be chosen later, and consider the subgrid $T_{h}$ of $T$ consisting of the rows with numbers $1, h+1,2 h+1, \ldots$. As each cell in row $i h+1$ contains $N_{1} 2^{i h}$ points, and there are $2^{t-1}$ cells in each row, the total number of points in row $i h+1$ is $X_{i}:=N_{1} 2^{t+i h-1}$.

Next we bound the number of points in row $i h+1$ covered by queries at levels $1, h+1, \ldots,(i-1) h+1$ of the query set $Q_{T}$. Level $j h+1$ contains $2^{j h}$ queries. Hence, the total number of queries at levels $1, h+1, \ldots,(i-1) h+1$ is $\sum_{j=0}^{i-1} 2^{j h}<2^{(i-1) h+1}$. The colour set of each such query contains at most $\alpha$ colours, and there are at most $B=N_{1}\left(2^{t}-1\right)$ points with the same colour. Hence, at most $Y_{i}:=\alpha N_{1} 2^{t+(i-1) h+1}=\left(4 \alpha / 2^{h}\right) X_{i}$ points have a colour belonging to the colour set of at least one of these queries and, thus, can be covered by these queries.

Now we choose $h=\lceil\log (16 \alpha)\rceil$. Then $Y_{i} \leq X_{i} / 4$. This implies that at least half of the cells in row $i h+1$ are $T_{h}$-exposed. Since this applies to all rows in $T_{h}$, there exists a column in $T_{h}$ at least half of whose cells are $T_{h}$-exposed. Let $Z$ be the set of $T_{h}$-exposed cells in this column. Since $T_{h}$ has $\lceil t / h\rceil=\Omega(t / \log \alpha)$ rows, $Z$ contains $\Omega(t / \log \alpha)$ cells, all of which are $Z$-exposed because $Z \subseteq T_{h}$.

By Lemma 4, there exists a sequence $Z=\left\langle c_{1}, c_{2}, \ldots, c_{s}\right\rangle$ of $s=\Omega(t / \log \alpha)$ $Z$-exposed cells in some column of $T$. Now, for all $1 \leq i \leq s$, we choose $p_{i}$ to be a $Z$-exposed point in cell $c_{i}$ with the minimum number of colours. It suffices to show that there exists an index $i$ such that $p_{i}$ has at least $s^{\varepsilon}$ colours, as this implies that all $Z$-exposed points in $c_{i}$ have at least this many colours and at least half the points in $c_{i}$ are $Z$-exposed. To prove this, we consider the point-query sequence $\left\langle\left(p_{1}, q_{1}\right),\left(p_{2}, q_{2}\right), \ldots,\left(p_{s}, q_{s}\right)\right\rangle$, where $p_{i}$ is chosen from the set of $Z$-exposed points in cell $c_{i}$ as just discussed, and $q_{i}:=q_{c_{i}}$, for all $1 \leq i \leq s$. The sequence $\left\langle\left(p_{1}, q_{1}\right),\left(p_{2}, q_{2}\right), \ldots,\left(p_{s}, q_{s}\right)\right\rangle$ has the following three properties.

(i) For $1 \leq i \leq j \leq s, C\left(p_{i}\right) \cap C\left(q_{j}\right) \neq \emptyset$ (because $\left.p_{i} \in q_{j}\right)$.

(ii) For $1 \leq j<i \leq s, C\left(p_{i}\right) \cap C\left(q_{j}\right)=\emptyset$ (because $p_{i}$ is exposed).

(iii) For $1 \leq j \leq s,\left|C\left(q_{j}\right)\right| \leq \alpha$.

We prove that any assignment of colour sets to the points and queries in $\left\langle\left(p_{1}, q_{1}\right)\right.$, $\left.\left(p_{2}, q_{2}\right), \ldots,\left(p_{s}, q_{s}\right)\right\rangle$ that satisfies these three properties also has the property that $\left|C\left(p_{i}\right)\right| \geq s^{1 / \alpha}=s^{\varepsilon}$, for some $1 \leq i \leq s$. We use the following terminology and notation.

We call an assignment of colour sets to the points and queries in the sequence $\left\langle\left(p_{1}, q_{1}\right),\left(p_{2}, q_{2}\right), \ldots,\left(p_{s}, q_{s}\right)\right\rangle$ a colouring. The colouring is proper if it satisfies conditions (i) and (ii). The colouring is an $(\alpha, \beta)$-colouring if each query is 
assigned at most $\alpha$ colours (condition (iii)) and each point is assigned at most $\beta$ colours. We say that a point-query sequence $\left\langle\left(p_{1}, q_{1}\right),\left(p_{2}, q_{2}\right), \ldots,\left(p_{s}, q_{s}\right)\right\rangle$ is $(\alpha, \beta)$-colourable if it has a proper $(\alpha, \beta)$-colouring. Finally, let $L(\alpha, \beta)$ be the maximum length $s$ such that the sequence $\left\langle\left(p_{1}, q_{1}\right),\left(p_{2}, q_{2}\right), \ldots,\left(p_{s}, q_{s}\right)\right\rangle$ is $(\alpha, \beta)$ colourable, and $\ell(\alpha, \beta):=L(\alpha, \beta-1)+1$ the minimum length $s$ such that the sequence $\left\langle\left(p_{1}, q_{1}\right),\left(p_{2}, q_{2}\right), \ldots,\left(p_{s}, q_{s}\right)\right\rangle$ is not $(\alpha, \beta-1)$-colourable. With this notation, our goal is to show that $\ell(\alpha, \beta) \leq \beta^{\alpha}$ because it implies that $\ell\left(\alpha, s^{1 / \alpha}\right) \leq s$, that is, a colouring of a point-query sequence $\left\langle\left(p_{1}, q_{1}\right),\left(p_{2}, q_{2}\right), \ldots,\left(p_{s}, q_{s}\right)\right\rangle$ of length $s$ that satisfies conditions (i)-(iii) assigns at least $s^{1 / \alpha}$ points to at least one point $p_{i}$.

Lemma 5 For $\alpha \geq 0$ and $\beta \geq 1, \ell(\alpha, \beta)=\left(\begin{array}{c}\alpha+\beta-1 \\ \alpha\end{array}\right) \leq \beta^{\alpha}$.

Proof First we prove that $\ell(\alpha, \beta)$ satisfies the recurrence relation

$$
\ell(\alpha, \beta)= \begin{cases}1 & \alpha=0 \text { or } \beta=1 \\ \ell(\alpha, \beta-1)+\ell(\alpha-1, \beta) & \alpha>0 \text { and } \beta>1 .\end{cases}
$$

The base case ( $\alpha=0$ or $\beta=1$ ) is fairly obvious: a point-query sequence of length 1 is neither $(0, \beta)$-colourable, for any $\beta$, nor $(\alpha, 0)$-colourable, for any $\alpha$, while a point-query sequence of length 0 is $(\alpha, \beta)$-colourable, for any $\alpha$ and $\beta$.

For the inductive step $(\alpha>0$ and $\beta>1)$, we prove that $L(\alpha, \beta)=\ell(\alpha, \beta)+$ $L(\alpha-1, \beta)$. This implies that

$$
\begin{aligned}
\ell(\alpha, \beta) & =L(\alpha, \beta-1)+1 \\
& =\ell(\alpha, \beta-1)+L(\alpha-1, \beta-1)+1 \\
& =\ell(\alpha, \beta-1)+(\ell(\alpha-1, \beta)-1)+1 \\
& =\ell(\alpha, \beta-1)+\ell(\alpha-1, \beta) .
\end{aligned}
$$

First we prove that $L(\alpha, \beta) \leq \ell(\alpha, \beta)+L(\alpha-1, \beta)$. To this end, we consider a point-query sequence $\left\langle\left(p_{1}, q_{1}\right),\left(p_{2}, q_{2}\right), \ldots,\left(p_{s}, q_{s}\right)\right\rangle$, where $s:=L(\alpha, \beta)$. Since $L(\alpha-1, \beta) \geq 0$, for all $\alpha$ and $\beta, s \leq \ell(\alpha, \beta)$ would immediately imply $s \leq \ell(\alpha, \beta)+$ $L(\alpha-1, \beta)$. Thus, we can assume that $s>\ell(\alpha, \beta)$. Let $s^{\prime}:=\ell(\alpha, \beta)$.

Now let $C$ be a proper $(\alpha, \beta)$-colouring of $\left\langle\left(p_{1}, q_{1}\right),\left(p_{2}, q_{2}\right), \ldots,\left(p_{s}, q_{s}\right)\right\rangle$. Since the restriction of the $C$ to the point-query sequence $\left\langle\left(p_{1}, q_{1}\right),\left(p_{2}, q_{2}\right), \ldots,\left(p_{s^{\prime}}, q_{s^{\prime}}\right)\right\rangle$ is a proper $(\alpha, \beta)$-colouring of this sequence, the definition of $\ell(\alpha, \beta)$ implies that there exists a point $p_{k}, 1 \leq k \leq s^{\prime}$, such that $\left|C\left(p_{k}\right)\right|=\beta$ and $C\left(p_{k}\right) \subseteq \bigcup_{j=1}^{s^{\prime}} C\left(q_{j}\right)$. We use this to construct a proper $(\alpha-1, \beta)$-colouring $C^{\prime}$ of the subsequence $\left\langle\left(p_{s^{\prime}+1}, q_{s^{\prime}+1}\right),\left(p_{s^{\prime}+2}, q_{s^{\prime}+2}\right), \ldots,\left(p_{s}, q_{s}\right)\right\rangle$, thereby showing that its length $s-s^{\prime}$ is at most $L(\alpha-1, \beta)$, which implies that $L(\alpha, \beta)=s=s^{\prime}+\left(s-s^{\prime}\right) \leq \ell(\alpha, \beta)+$ $L(\alpha-1, \beta)$.

To obtain such a colouring $C^{\prime}$, we define $C^{\prime}\left(p_{i}\right)=C\left(p_{i}\right)$ and $C^{\prime}\left(q_{i}\right)=C\left(q_{i}\right) \backslash$ $C\left(p_{k}\right)$, for all $s^{\prime}<i \leq s$. By property (i) of $C, C\left(q_{i}\right) \cap C\left(p_{k}\right) \neq \emptyset$, for $s^{\prime}<i \leq s$ and, hence, $\left|C^{\prime}\left(q_{i}\right)\right|<\left|C\left(q_{i}\right)\right| \leq \alpha$, while $\left|C^{\prime}\left(p_{i}\right)\right|=\left|C\left(p_{i}\right)\right| \leq \beta$. Thus, $C^{\prime}$ is an $(\alpha-1, \beta)$-colouring of the sequence $\left\langle\left(p_{s^{\prime}+1}, q_{s^{\prime}+1}\right),\left(p_{s^{\prime}+2}, q_{s^{\prime}+2}\right), \ldots,\left(p_{s}, q_{s}\right)\right\rangle$. Next we show that $C^{\prime}$ is proper. 
First observe that, for $s^{\prime}<j<i \leq s, C^{\prime}\left(p_{i}\right) \cap C^{\prime}\left(q_{j}\right) \subseteq C\left(p_{i}\right) \cap C\left(q_{j}\right)=\emptyset$, by property (ii) of $C$. Hence, $C^{\prime}$ satisfies property (ii).

For $s^{\prime}<i \leq j \leq s$, we have $C\left(p_{i}\right) \cap C\left(p_{k}\right) \subseteq C\left(p_{i}\right) \cap \bigcup_{h=1}^{s^{\prime}} C\left(q_{h}\right)=\emptyset$, by property (ii) of $C$. Hence, $C^{\prime}\left(p_{i}\right) \cap C^{\prime}\left(q_{j}\right)=C\left(p_{i}\right) \cap\left(C\left(q_{j}\right) \backslash C\left(p_{k}\right)\right)=C\left(p_{i}\right) \cap C\left(q_{j}\right)$ $\neq \emptyset$, by property (i) of $C$. Thus, $C^{\prime}$ satisfies property (i). This concludes the proof of the inequality $L(\alpha, \beta) \leq \ell(\alpha, \beta)+L(\alpha-1, \beta)$.

Next we prove that a point-query sequence $\left\langle\left(p_{1}, q_{1}\right),\left(p_{2}, q_{2}\right), \ldots,\left(p_{s}, q_{s}\right)\right\rangle$ of length $s:=\ell(\alpha, \beta)+L(\alpha-1, \beta)=L(\alpha, \beta-1)+L(\alpha-1, \beta)+1$ is $(\alpha, \beta)$ colourable. This proves that $L(\alpha, \beta) \geq \ell(\alpha, \beta)+L(\alpha-1, \beta)$.

We divide the sequence into three subsequences $\left\langle\left(p_{1}, q_{1}\right),\left(p_{2}, q_{2}\right), \ldots,\left(p_{s_{1}}, q_{s_{1}}\right)\right\rangle$, $\left\langle\left(p_{s_{1}+1}, q_{s_{1}+1}\right)\right\rangle$, and $\left\langle\left(p_{s_{1}+2}, q_{s_{1}+2}\right),\left(p_{s_{1}+3}, q_{s_{1}+3}\right), \ldots,\left(p_{s}, q_{s}\right)\right\rangle$, where $s_{1}:=L(\alpha$, $\beta-1)$ and $s_{3}:=s-s_{1}-1=L(\alpha-1, \beta)$. By the choice of $s_{1}$ and $s_{3}$, the sequence $\left\langle\left(p_{1}, q_{1}\right),\left(p_{2}, q_{2}\right), \ldots,\left(p_{s_{1}}, q_{s_{1}}\right)\right\rangle$ has a proper $(\alpha, \beta-1)$-colouring $C_{1}$, and the sequence $\left\langle\left(p_{s_{1}+2}, q_{s_{1}+2}\right),\left(p_{s_{1}+3}, q_{s_{1}+3}\right), \ldots,\left(p_{s}, q_{s}\right)\right\rangle$ has a proper $(\alpha-1, \beta)$ colouring $C_{3}$. We can assume that the two colourings use different colours. We define a colouring $C$ of the sequence $\left\langle\left(p_{1}, q_{1}\right),\left(p_{2}, q_{2}\right), \ldots,\left(p_{s}, q_{s}\right)\right\rangle$ as

$$
C\left(p_{i}\right)= \begin{cases}C_{1}\left(p_{i}\right) \cup\{\gamma\} & 1 \leq i \leq s_{1} \\ \{\gamma\} & i=s_{1}+1 \\ C_{3}\left(p_{i}\right) & s_{1}+2 \leq i \leq s\end{cases}
$$

and

$$
C\left(q_{i}\right)= \begin{cases}C_{1}\left(q_{i}\right) & 1 \leq i \leq s_{1} \\ \{\gamma\} & i=s_{1}+1 \\ C_{3}\left(q_{i}\right) \cup\{\gamma\} & s_{1}+2 \leq i \leq s,\end{cases}
$$

where $\gamma$ is a new colour not used by either $C_{1}$ or $C_{3}$. Since $C_{1}$ is an $(\alpha, \beta-1)$ colouring of the sequence $\left\langle\left(p_{1}, q_{1}\right),\left(p_{2}, q_{2}\right), \ldots,\left(p_{s_{1}}, q_{s_{1}}\right)\right\rangle$ and $C_{3}$ is an $(\alpha-1, \beta)$ colouring of $\left\langle\left(p_{s_{1}+2}, q_{s_{1}+2}\right),\left(p_{s_{1}+3}, q_{s_{1}+3}\right), \ldots,\left(p_{s}, q_{s}\right)\right\rangle, C$ is an $(\alpha, \beta)$-colouring of $\left\langle\left(p_{1}, q_{1}\right),\left(p_{2}, q_{2}\right), \ldots,\left(p_{s}, q_{s}\right)\right\rangle$. Next we show that $C$ is proper.

First consider property (i). For a point $p_{i}$ and a query $q_{j}$ with $1 \leq i \leq j \leq s$, we distinguish three cases. If $j \leq s_{1}$, then $C\left(p_{i}\right) \supset C_{1}\left(p_{i}\right)$ and $C\left(q_{j}\right)=C_{1}\left(q_{j}\right)$. Hence, since $C_{1}\left(p_{i}\right) \cap C_{1}\left(q_{j}\right) \neq \varnothing$ (by property (i) of the colouring $\left.C_{1}\right)$, we have $C\left(p_{i}\right) \cap C\left(q_{j}\right) \neq \emptyset$. If $i \leq s_{1}+1 \leq j$, we have $\gamma \in C\left(p_{i}\right) \cap C\left(q_{j}\right)$. If $s_{1}+1<i$, then $C\left(p_{i}\right)=C_{3}\left(p_{i}\right)$ and $C\left(q_{j}\right) \supset C_{3}\left(q_{j}\right)$. Hence, since $C_{3}\left(p_{i}\right) \cap C_{3}\left(q_{j}\right) \neq \varnothing$ (by property (i) of the colouring $\left.C_{3}\right)$, we have $C\left(p_{i}\right) \cap C\left(q_{j}\right) \neq \emptyset$.

Next we verify property (ii). Consider a point $p_{i}$ and a query $q_{j}$ with $1 \leq j<$ $i \leq s$. If $i \leq s_{1}$, we have $C\left(p_{i}\right) \cap C\left(q_{j}\right)=\emptyset$ because $C_{1}\left(p_{i}\right) \cap C\left(q_{j}\right)=C_{1}\left(p_{i}\right) \cap$ $C_{1}\left(q_{j}\right)=\emptyset$ (by property (ii) of the colouring $\left.C_{1}\right)$ and $\gamma \notin C\left(q_{j}\right)$. If $j \leq s_{1}<i$, we have $C\left(q_{j}\right) \cap C\left(p_{i}\right)=\emptyset$ because $\gamma \notin C\left(q_{j}\right)$ and colourings $C_{1}$ and $C_{3}$ use different colours. Finally, if $s_{1}+1 \leq j$, then $C\left(p_{i}\right) \cap C\left(q_{j}\right)=\emptyset$ because $C\left(p_{i}\right) \cap C_{3}\left(q_{j}\right)=$ $C_{3}\left(p_{i}\right) \cap C_{3}\left(q_{j}\right)=\emptyset$ (by property (ii) of the colouring $\left.C_{3}\right)$ and $\gamma \notin C\left(p_{i}\right)$.

This shows that $C$ is a proper $(\alpha, \beta)$-colouring of the sequence $\left\langle\left(p_{1}, q_{1}\right),\left(p_{2}, q_{2}\right)\right.$, $\left.\ldots,\left(p_{s}, q_{s}\right)\right\rangle$ and, hence, that $\ell(\alpha, \beta)+L(\alpha-1, \beta)=s \leq L(\alpha, \beta)$. 
Having established the correctness of (1), it remains to derive a closed form for $\ell(\alpha, \beta)$. We do this using induction. For the base case, we have $\ell(0, \beta)=1=\left(\begin{array}{c}\beta-1 \\ 0\end{array}\right)$ and $\ell(\alpha, 1)=1=\left(\begin{array}{l}\alpha \\ \alpha\end{array}\right)$. For the inductive step, we obtain

$$
\begin{aligned}
\ell(\alpha, \beta) & =\ell(\alpha, \beta-1)+\ell(\alpha-1, \beta) \\
& =\left(\begin{array}{c}
\alpha+\beta-2 \\
\alpha
\end{array}\right)+\left(\begin{array}{c}
\alpha+\beta-2 \\
\alpha-1
\end{array}\right) \\
& =\left(\begin{array}{c}
\alpha+\beta-1 \\
\alpha
\end{array}\right) .
\end{aligned}
$$

This finishes the proof.

To summarize, Lemma 1 is established by invoking Lemma 4 to prove that there exists a set $Z$ of $s=\Omega(t / \log \alpha) Z$-exposed cells in $T$. Using Lemma 5, we show that the $Z$-exposed point with the minimum number of colours in at least one of these cells has at least $s^{\varepsilon}=\Omega\left(t^{\varepsilon}\right)$ colours, for $\varepsilon=1 / \alpha$ and $\alpha=f(2,1)$. Thus, every $Z$-exposed point in this cell has at least $\Omega\left(t^{\varepsilon}\right)$ colours. Since at least half the points in a $Z$-exposed cell are $Z$-exposed, at least half the points in this cell have $\Omega\left(t^{\varepsilon}\right)$ colours. The same argument can be applied to every subgrid in the recursive construction of the point set $S$, which implies that every $f$-efficient indexing scheme for the workload $\mathcal{W}$ satisfies Lemma 3 with $m=\Omega\left(t^{\varepsilon}\right)$, which proves Lemma 1 for $\delta=1 / 2$. By Lemma 2, this implies Lemma 1 for any $0<\delta \leq 1 / 2$.

\section{Tightness of the Lower Bound}

In this section, we show that the lower bound of Lemma 1 is tight for the workload $\mathcal{W}=(S, Q, \mathcal{B})$ we constructed in the previous section, up to the dependence of $\varepsilon$ on $f(\cdot, \cdot)$.

Theorem 2 Let $f(\cdot, \cdot)$ be a monotonically increasing function such that $f(x, y) \geq$ $\lceil y\rceil$, and let $\alpha:=f(2,1)$. There exists a g-efficient indexing scheme $\mathcal{I}$ for $\mathcal{W}$ of size $\mathrm{O}\left(N(\log \log N)^{1 / \alpha}\right)$, for some function $g(\cdot, \cdot)$ that satisfies $g(x, y)=\mathrm{O}(f(x, y))$.

The condition that $f(x, y) \geq\lceil y\rceil$ is not a restriction because every function $f(\cdot, \cdot)$ such that there exists an $f$-efficient indexing scheme for $\mathcal{W}$ satisfies this condition. To prove Theorem 2, we define a linear layout of the points in $S$ that stores each point $\mathrm{O}\left(t^{1 / \alpha}\right)$ times and such that every query $q \in Q$ can be decomposed into $\mathrm{O}(\alpha+1)$ contiguous subsequences of this linear layout. By partitioning the layout into blocks of size $B$, for any $B$, we obtain a $B$-cover of $S$ that can cover every query in $Q$ with $\mathrm{O}(\alpha+1+K / B)=\mathrm{O}\left(f\left(\log _{B} N, K / B\right)\right)$ blocks because $\alpha=f(2,1)=\mathrm{O}(1)$ and $f\left(\log _{B} N, K / B\right) \geq\lceil K / B\rceil$. In particular, the $B_{i}$-covers obtained in this fashion, for all $B_{i} \in \mathcal{B}$, form a $g$-efficient indexing scheme of size $\mathrm{O}\left(N t^{1 / \alpha}\right)=\mathrm{O}\left(N(\log \log N)^{1 / \alpha}\right)$ for $\mathcal{W}$, where $g(x, y)=\mathrm{O}(f(x, y))$. 
The layout To construct the layout of the points in $S$, let $\beta$ be the smallest integer such that $\ell(\alpha, \beta+1) \geq t$; that is, $\beta=\mathrm{O}\left(t^{1 / \alpha}\right)=\mathrm{O}\left((\log \log N)^{1 / \alpha}\right)$. To simplify the argument, we can assume that $\ell(\alpha, \beta+1)=t$, which we can achieve by padding each grid with empty rows at the bottom. Our layout consists of $\beta$ copies of $S$, which immediately implies that the layout uses $N \beta=\mathrm{O}\left(N(\log \log N)^{1 / \alpha}\right)$ space.

In the first copy, we divide the rows of the top-level grid $T$ into $\alpha+1$ groups $G_{0}, G_{1}, \ldots, G_{\alpha}$, ordered from top to bottom. The size of group $G_{i}$ is $\ell(\alpha-i, \beta)$. The cells in each group are laid out in column-major order. This is illustrated in Fig. 3a. Note that the groups $G_{0}, G_{1}, \ldots, G_{\alpha}$ cover all the rows of $T$ because $t=\ell(\alpha, \beta+1)=\sum_{i=0}^{\alpha} \ell(i, \beta)$; the second equality follows from the classic equality $\left(\begin{array}{l}n+1 \\ m+1\end{array}\right)=\sum_{i=0}^{n}\left(\begin{array}{l}i \\ m\end{array}\right)$. To complete the layout of the points in the first copy of $S$, we have to determine the order in which to arrange the points in each cell of $T$. Since each cell of $T$ is divided recursively into $t \times 2^{t-1}$ subgrids, we can divide the rows of each such subgrid $T^{\prime}$ into groups $G_{0}^{\prime}, G_{1}^{\prime}, \ldots, G_{\alpha}^{\prime}$ in the same fashion as just described and lay out the cells in each group in column-major order. This continues recursively until we reach subgrids $T^{\prime}$ whose cells have not been divided further in the construction of the point set $S$. For each such subgrid, we arrange the points in each cell in an arbitrary order.

For $1<k \leq \beta$, the layout of the points in the $k$ th copy of $S$ is obtained by dividing the groups used to define the $(k-1)$ st copy into subgroups and laying out the cells in each subgroup in column-major order. In particular, a group $G$ in the $(k-1)$ st copy consisting of $\ell\left(\alpha^{\prime}, \beta-k+2\right)$ rows is divided into $\alpha^{\prime}+1$ subgroups $G_{0}, G_{1}, \ldots, G_{\alpha^{\prime}}$, with $G_{i}$ covering $\ell(i, \beta-k+1)$ rows. This is illustrated in Fig. 3a for the second copy of $S$. Similar to the argument for the first copy of $S$, we have $\ell\left(\alpha^{\prime}, \beta-k+2\right)=$ $\sum_{i=0}^{\alpha^{\prime}} \ell(i, \beta-k+1)$, that is, the subgroups cover the rows of $G$ exactly. To determine the order in which to lay out the points in each cell of $T$, we apply the same refinement process to the groups in each subgrid $T^{\prime}$ in the recursive construction of $S$.

By continuing in this fashion until $k=\beta$, we obtain that every group in the $\beta$ th copy of $S$ consists of $\ell\left(\alpha^{\prime}, 1\right)=1$ rows, for some $\alpha^{\prime}$. Hence, the $\beta$ th copy of $S$ stores

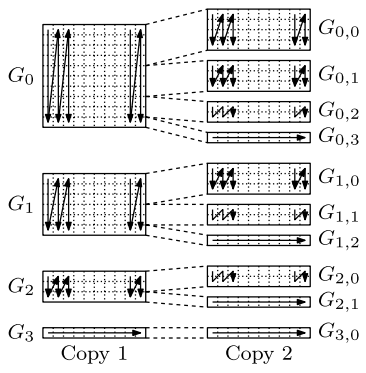

(a)

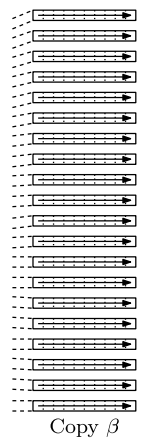

Fig. 3 (a) An $\mathrm{O}\left(N(\log \log N)^{1 / \alpha}\right)$-space layout for the point set $S$ (layout shown for $\alpha=3$ ). (b) Answering a query on the first two levels of the layout. The dark portion of the query in each copy of $S$ forms a contiguous subsequence of the column-major layout of the respective group. The light portion is answered using subsequent copies of $S$ 
the cells in each row of $T$ (and of any subgrid $T^{\prime}$ ) in $x$-sorted order. This is illustrated on the right of Fig. 3a.

Answering queries Since the layout arranges the cells of each subgrid $T^{\prime}$ in the recursive construction of $S$ in the same fashion as the cells of $T$, it suffices to prove that any query $q \in Q_{T}$ can be partitioned into at most $\alpha+1$ contiguous subsequences in the layout.

Assume the query $q$ is at the $i$ th level of $Q_{T}$, that is, its bottom boundary coincides with the bottom boundary of the $i$ th row of $T$. If this row is the bottom-most row of some group $G_{j_{1}}$ in the first copy of $S$, observe that the points in $q$ that belong to any group $G_{j}, 0 \leq j \leq j_{1}$, are stored contiguously in the first copy of $S$. Hence, the points in $q$ are divided into $j_{1}+1$ contiguous subsequences.

If row $i$ belongs to group $G_{j_{1}}$ but is not its bottom row, we use the first copy of $S$ to report all points of $q$ that belong to groups $G_{0}, G_{1}, \ldots, G_{j_{1}-1}$ and use subsequent copies of $S$ to report all points in $q$ that belong to $G_{j_{1}}$. We say that group $G_{j_{1}}$ is the partial group of $q$ in the first copy of $S$. By the arguments in the previous paragraph, the points of $q$ that belong to groups $G_{0}, G_{1}, \ldots, G_{j_{1}-1}$ form $j_{1}$ contiguous subsequences in the first copy of $S$.

To see how subsequent copies of $S$ are used to report the remaining points of $q$, consider the $k$ th copy and let $G$ be the partial group of $q$ in the $(k-1)$ st copy. This group has $\ell\left(\alpha^{\prime}, \beta-k+2\right)$ rows, for some $\alpha^{\prime}$. Then we consider the subgroup $G_{j_{k}}$ of $G$ containing row $i$. As for the first copy of $S$, if row $i$ is the bottom-most row of group $G_{j_{k}}$, we use groups $G_{0}, G_{1}, \ldots, G_{j_{k}}$ to report all points in $G$ that belong to $q$. These points are stored in $j_{k}+1$ contiguous subsequences of the $k$ th copy of $S$. If row $i$ is not the bottom-most row of group $G_{j_{k}}$, then $G_{j_{k}}$ is once again a partial group of $q$, and we report only those points in $q$ that belong to $G_{0}, G_{1}, \ldots, G_{j_{k}-1}$ using the $k$ th copy of $S$, and use subsequent copies of $S$ to report the points in $q$ that belong to $G_{j_{k}}$. Figure 3 b illustrates this recursive partitioning of query $q$ for the first two copies of $S$. Note that this procedure terminates at the latest when reaching the last copy of $S$ because this copy stores all rows in $x$-sorted order, that is, the group $G_{j_{\beta}}$ cannot be partial.

It remains to bound the number of contiguous subsequences into which the points of $q$ are divided by this procedure. If the procedure terminates after inspecting the first $s$ copies of $S$, the points in $q$ are divided into $1+\sum_{k=1}^{s} j_{k}$ contiguous subsequences, $j_{k}$ in the $k$ th copy of $S$, for $1 \leq k<s$, and $j_{s}+1$ in the $s$ th copy. For all $1<k \leq s$, if the partial group $G$ of $q$ in the $(k-1)$ st copy of $S$ has $\ell\left(\alpha^{\prime}, \beta-i+2\right)$ rows, then $j_{k} \leq \alpha^{\prime}$ and $G_{j_{k}}$ has $\ell\left(\alpha^{\prime}-j_{k}, \beta-i+1\right)$ rows. Since $j_{1} \leq \alpha$, this implies that $1+\sum_{k=1}^{s} j_{k} \leq \alpha+1$ using a simple inductive argument, that is, each query $q \in Q$ is partitioned into at most $\alpha+1$ contiguous subsequences of the layout.

\section{Further Lower Bounds}

In this section, we show that the proof technique from Sect. 3 can be used to obtain the same lower bound as in Theorem 1 for other range searching problems and to obtain a lower bound on the space consumption of cache-oblivious persistent B-trees with optimal 1-d range queries. The lower bounds in Sect. 5.1, for 3-d dominance reporting and persistent B-trees, are obtained using direct reductions from three-sided 
range reporting. In Sect. 5.2, we prove the same lower bound for 3-d halfspace range reporting, but this requires more care, as there is no direct reduction from three-sided range reporting to 3-d halfspace range reporting. Finally, in Sect. 5.3, we show that all lower bounds proved in Sects. 3, 5.1, and 5.2 also hold for the expected size of Las-Vegas-type data structures that achieve an expected query bound of at most $f\left(\log _{B} N, K / B\right)$ block transfers.

\subsection{3-D Dominance Reporting and Persistent B-Trees}

The first result in this section is a lower bound on the space consumption of any cache-oblivious data structure for 3-d dominance reporting, as summarized in the following theorem.

Theorem 3 Let $f(\cdot, \cdot)$ be a monotonically increasing function, and $0<\delta \leq 1 / 2$ a constant. Any cache-oblivious data structure capable of answering 3-d dominance reporting queries using at most $f\left(\log _{B} N, K / B\right)$ block transfers in the worst case, for every block size $B \leq N^{2 \delta}$, must use $\Omega\left(N(\log \log N)^{\varepsilon}\right)$ space, where $\varepsilon=1 / f\left(\delta^{-1}, 1\right)$.

Proof Just as Theorem 1, this result follows if we can show that the workload $\mathcal{W}=$ $(S, Q, \mathcal{B})$ constructed in Sect. 3 to prove Lemma 1 is realizable as a 3-d dominance reporting problem. We do this using a simple geometric transformation. We map each input point $p=\left(x_{p}, y_{p}\right)$ in $S$ to the point $\phi(p)=\left(-x_{p}, x_{p},-y_{p}\right)$ in $\mathbb{R}^{3}$, and every three-sided query range $q=[l, r] \times[b,+\infty)$ in $Q$ to the query range $\phi(q)=$ $(-\infty,-l] \times(-\infty, r] \times(-\infty,-b]$. A point $p \in S$ belongs to a three-sided query range $q \in Q$ if and only if $\phi(p)$ belongs to $\phi(q)$, that is, the mapping provides a realization of $\mathcal{W}$ as a 3 -d dominance reporting problem.

A similar reduction shows the following result.

Theorem 4 Let $f(\cdot, \cdot)$ be a monotonically increasing function, and $0<\delta \leq 1 / 2$ a constant. Any (partially) persistent cache-oblivious B-tree capable of answering 1-d range reporting queries on any previous version of the tree using at most $f\left(\log _{B} N, K / B\right)$ block transfers in the worst case, for every block size $B \leq N^{2 \delta}$, must use $\Omega\left(N(\log \log N)^{\varepsilon}\right)$ space to represent a sequence of $N$ update operations, where $\varepsilon=1 / f\left(\delta^{-1}, 1\right)$.

Proof Again, we need to show that the workload $\mathcal{W}=(S, Q, \mathcal{B})$ from Sect. 3 is realizable in the framework of 1-d range reporting queries on persistent B-trees. We say that $\mathcal{W}$ is realizable as a persistent 1-d range reporting problem if there exists a sequence of insertions and deletions of the elements in $S$ and a mapping of the elements in $S$ to points on the real line such that, for every query $q \in Q$, there exists an interval $[l, r]$ and an integer $t$ with the property that a 1-d range reporting query with interval $[l, r]$ after the $t$ th operation in the update sequence reports exactly the points in $q$.

To obtain such a realization of the workload $\mathcal{W}$, we map each point $p=\left(x_{p}, y_{p}\right)$ in $S$ to the point $x_{p}$ on the real line and define an update sequence that inserts the 
points in $S$ by decreasing $y$-coordinates. For a query $q=[l, r] \times[b,+\infty)$ in $Q$, let $t$ be the number of points $p \in S$ with $y_{p} \geq b$. Then a 1-d range query with interval $[l, r]$ after the $t$ th insertion reports exactly the points in $q$. Thus, $\mathcal{W}$ is realizable as a persistent 1-d range reporting problem.

\subsection{Halfspace Range Reporting in Three Dimensions}

In this section, we prove the same lower bound as in Theorem 1 for 3 -d halfspace range reporting.

Theorem 5 Let $f(\cdot, \cdot)$ be a monotonically increasing function, and $0<\delta \leq 1 / 2$ a constant. Any cache-oblivious data structure capable of answering 3-d halfspace range reporting queries using at most $f\left(\log _{B} N, K / B\right)$ block transfers in the worst case, for every block size $B \leq N^{2 \delta}$, must use $\Omega\left(N(\log \log N)^{\varepsilon}\right)$ space, where $\varepsilon=1 / f\left(\delta^{-1}, 1\right)$.

As in the proofs of Theorems 3 and 4, we need to show that the workload $\mathcal{W}=(S, Q, \mathcal{B})$ from Sect. 3 is realizable as a 3 -d halfspace range reporting problem. This requires more care than for 3 -d dominance reporting and persistent $1-\mathrm{d}$ range reporting. We begin with a reduction from 2 -d parabolic range reporting to 3 -d halfspace range reporting (see, e.g., [2]). A parabolic range reporting query is defined by a parabola $y=a(x-b)^{2}+c$, for $a \geq 0$, and asks to report all points $p=\left(x_{p}, y_{p}\right)$ in $S$ that satisfy $y_{p} \geq a\left(x_{p}-b\right)^{2}+c$. The reduction from [2] maps each point $p$ in $\mathbb{R}^{2}$ to a point $\psi(p)$ in $\mathbb{R}^{3}$ and each parabola $q$ to a halfspace $\psi(q)$ such that $p \in q$ if and only if $\psi(p) \in \psi(q)$. Thus, every workload realizable as a 2-d parabolic range reporting problem is also realizable as a 3-d halfspace range reporting problem. In particular, it suffices to show that $\mathcal{W}$ is realizable as a 2-d parabolic range reporting problem. We do this by distorting the point set $S$ so that there exists a parabolic query range $\phi(q)$ for every three-sided query $q \in Q$ with the property that $q$ and $\phi(q)$ contain the same points in $S$.

We follow the recursive construction of $S$ and $Q$ from Sect. 3 and, for every subgrid $T^{\prime}$ in the construction, replace the three-sided queries in $Q_{T^{\prime}}$ with parabolic ones as shown in Fig. 4a. If we embed the subgrids of $T^{\prime}$ inside the white squares in the figure, each query outputs the same set of points in $T^{\prime}$ as its corresponding threesided query. However, we also have to ensure that the queries in $Q_{T^{\prime}}$ output only points from $T^{\prime}$. For three-sided queries, we achieved this by ensuring that there are no points in the $x$-range of $T^{\prime}$ but outside its $y$-range. As shown in Fig. $4 \mathrm{~b}$, this is not sufficient for parabolic queries, as parabolic queries over $T^{\prime}$ cannot be confined to the $x$-range of $T^{\prime}$. Instead, we use the following construction, which extends a construction from [2].

Given a grid cell and a subgrid $T^{\prime}$ to be embedded inside this cell, we use $G$ to denote the portion of the cell where the points in $T^{\prime}$ are to be placed (the white squares in Fig. 4a). We call $G$ the grid box of $T^{\prime}$. Every parabolic query over $T^{\prime}$ is guaranteed to output only points from $T^{\prime}$ if it leaves the $x$-range of $G$ only above the top boundary of the top-level grid $T$. We represent this using a column box $C$ that shares its left, right, and bottom boundaries with $G$ and whose top boundary is the 


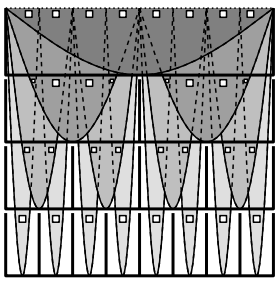

(a)

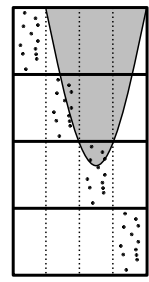

(b)

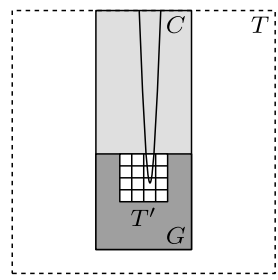

(c)

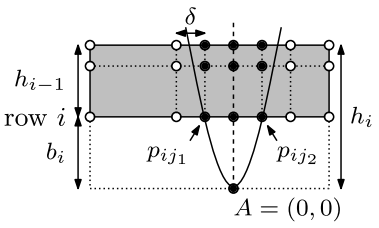

(d)

Fig. 4 (a) Replacing three-sided queries with parabolic ones. The white squares are the areas where the subgrids in each grid cell are to be placed. (b) A naively constructed query in the subgrid in cell $T_{3,6}$ also reports points in other cells (e.g., $T_{2,6}$ ). (c) Placement of a subgrid within a grid box $G$ that is nested inside a column box $C$. (d) Incremental embedding of a subgrid $T^{\prime}$ inside a grid box

top boundary of $T$; see Fig. 4c. The parabolic queries over $T^{\prime}$ must intersect only the top boundary edge of $C$. Once we have obtained an embedding of $T^{\prime}$ inside $G$ and a set of parabolic queries over $T^{\prime}$ that output the same set of points as the threesided queries in $Q_{T^{\prime}}$ and intersect only the top boundary of $C$, we can define grid and column boxes for the subgrids of $T^{\prime}$ as above and apply this construction recursively. Thus, to prove that $\mathcal{W}$ is realizable as a $2-\mathrm{d}$ parabolic range reporting problem, it suffices to prove that, given any grid box $G$ and any column box $C \supseteq G$, we can embed $T^{\prime}$ inside $G$ and construct appropriate parabolic queries over $T^{\prime}$ that intersect only the top boundary of $C$.

Lemma 6 Given a column box $C$ and a grid box $G \subseteq C$, a subgrid $T^{\prime}$ can be embedded inside $G$ so that, for every three-sided range reporting query $q \in Q_{T^{\prime}}$, there exists a parabolic range reporting query $q^{\prime}$ that reports the same set of points in $T^{\prime}$ as $q$ and intersects only the top boundary of $C$.

Proof For the sake of this proof, we can assume that each grid cell of $T^{\prime}$ contains exactly one point, as we can embed the subgrid represented by each such point in a sufficiently small neighbourhood of the point without altering the properties of the construction. We can further assume that the bottom boundaries of $C$ and $G$ coincide, that $C$ is twice as wide as $G$, and that $G$ is horizontally centred inside $C$, as this can be enforced by shrinking $G$ and $C$ appropriately without relaxing the constraints placed by these two boxes on the embedding of $T^{\prime}$ and on the queries constructed over $T^{\prime}$. We denote the widths and heights of $G$ and $C$ by $w_{G}, w_{C}, h_{G}$, and $h_{C}$, respectively.

We construct $T^{\prime}$ row by row, placing the points in each row at the same $y$-coordinate and spacing them evenly in $x$-direction. The top row of $T^{\prime}$ coincides with the top boundary of $G$, and we centre $T^{\prime}$ horizontally in $G$. The points in each row have distance $\delta>0$ between every pair of consecutive points. After placing the points in the $i$ th row, we construct parabolic queries for all queries at level $i$ in $Q_{T^{\prime}}$ that output exactly those points in rows 1 through $i$ contained in their three-sided counterparts in $Q_{T^{\prime}}$. The next row of points is then placed infinitesimally below the horizontal line through the apexes of these level- $i$ queries. This is illustrated in Fig. 4d.

Once we have placed the points of all rows of $T^{\prime}$ in this fashion, we obtain an embedding of $T^{\prime}$ inside a box of width $\delta\left(2^{t-1}-1\right)$ and height $h_{t}$. We derive a bound on 
$h_{t}$ as a function of $\delta$. By choosing $\delta$ small enough, we can ensure that the constructed box (and, hence, $T^{\prime}$ ) is completely contained in $G$.

To discuss this construction in detail, consider the construction of the $i$ th row. Let $B_{i-1}$ be the smallest box containing all points already placed in rows 1 and $i-1$ and such that its bottom boundary passes through the apexes of all level- $(i-1)$ queries we have just constructed. The width of $B_{i-1}$ is $\delta\left(2^{t-1}-1\right)$, and we denote its height by $h_{i-1}$. For $i=1$, we assume that $h_{0}=0$, that is, that $B_{0}$ is a line segment contained in the top boundary of $G$. To construct the $i$ th row of $T^{\prime}$, we evenly distribute $2^{t-1}$ points along a horizontal line segment of the same width as $B_{i-1}$ and infinitesimally below the bottom boundary of $B_{i-1}$. This ensures that no point in the $i$ th row is contained in any query at a level less than $i$. To construct the queries at the $i$ th level, we observe that each such query $q^{\prime}$ has to output points $p_{i^{\prime} j^{\prime}}$ with $1 \leq i^{\prime} \leq i$ and $\left(k^{\prime}-1\right) 2^{t-i}<j^{\prime} \leq k^{\prime} 2^{t-i}$, for some $0<k^{\prime} \leq 2^{i-1}$, where $p_{i j}$ denotes the $j$ th point in the $i$ th row. For ease of notation, let $j_{1}=\left(k^{\prime}-1\right) 2^{t-i}+1$ and $j_{2}=k^{\prime} 2^{t-i}$. We construct the parabola $q^{\prime}$ so that it passes through points $p_{i j_{1}}$ and $p_{i j_{2}}$ and such that points $p_{1, j_{1}-1}$ and $p_{1, j_{2}+1}$, as well as the two top corners of $C$, lie below $q^{\prime}$. This ensures that $q^{\prime}$ outputs exactly the desired set of points and intersects only the top boundary edge of $C$.

To obtain a bound on the height $h_{t}$ of the final box $B_{t}$, we first derive a bound on the distance $b_{i}$ between the apex $A$ of $q^{\prime}$ and the $i$ th row of points, for all $1 \leq i \leq t$, and then use the recurrence $h_{i}>h_{i-1}+b_{i}$. To ease the exposition, we assume that the apex $A$ of $q^{\prime}$ is at the origin, so that $q^{\prime}$ is given by an equation of the form $y=a_{i} x^{2}$. Let $d_{i}=\delta\left(2^{t-i}-1\right) / 2$ be half the distance between points $p_{i j_{1}}$ and $p_{i j_{2}}$. Since $A$ has distance $b_{i}$ from the $i$ th row of points and $q^{\prime}$ passes through points $p_{i j_{1}}$ and $p_{i j_{2}}, q^{\prime}$ must satisfy

$$
b_{i}=a_{i} d_{i}^{2} .
$$

In order for points $p_{1, j_{1}-1}$ and $p_{1, j_{2}+1}$ to lie below $q^{\prime}, q^{\prime}$ must satisfy

$$
h_{i-1}+b_{i}<a_{i}\left(d_{i}+\delta\right)^{2} \text {, }
$$

as the distance of $A$ from the first row is infinitesimally greater than $h_{i-1}+b_{i}$ and the distance between two neighbouring columns of $T^{\prime}$ is $\delta$. Finally, if we can satisfy (2) and (3) while placing $A$ inside $G$, then $q^{\prime}$ intersects only the top boundary edge of $C$ if

$$
h_{C}<a_{i} \frac{w_{G}^{2}}{4},
$$

as the height of $C$ is $h_{C}$ and the distance between $G$ and either of the two vertical boundary edges of $C$ is $w_{G} / 2$.

By substituting (2) into (3) and rearranging the result, we obtain that (3) holds if

$$
a_{i}>\frac{h_{i-1}}{2 d_{i} \delta+\delta^{2}} .
$$

Inequalities (4) and (5) are both satisfied if

$$
a_{i}>\frac{h_{i-1}}{2 d_{i} \delta+\delta^{2}}+\frac{4 h_{C}}{w_{G}^{2}},
$$


which gives

$$
b_{i}>\left(\frac{h_{i-1}}{2 d_{i} \delta+\delta^{2}}+\frac{4 h_{C}}{w_{G}^{2}}\right) d_{i}^{2}
$$

by substituting (6) into (2). By using the equation $d_{i}=\delta\left(2^{t-i}-1\right) / 2$ and simplifying appropriately, we obtain that (7) holds if

$$
b_{i}>2^{t-i-2} h_{i-1}+\frac{4^{t-i} \delta^{2} h_{C}}{w_{G}^{2}} .
$$

By substituting this into the recurrence $h_{i}>h_{i-1}+b_{i}$, we obtain that we can satisfy (8) as long as

$$
h_{i}>h_{i-1}\left(1+2^{t-i-2}\right)+\frac{4^{t-i} \delta^{2} h_{C}}{w_{G}^{2}},
$$

which holds if we set $h_{0}=0$ and $h_{i}=4^{i t} \delta^{2} h_{C} / w_{G}^{2}$ and as long as $t \geq 2$. Thus, by choosing $\delta$ no greater than $\min \left(w_{G} / 2^{t}, \sqrt{h_{G} w_{G}^{2} /\left(4^{t^{2}} h_{C}\right)}\right)$, we can ensure that all points of $T^{\prime}$ lie inside $G$, and that all parabolas in $Q_{T^{\prime}}$ have their apexes inside $G$, output the same set of points as their corresponding three-sided queries, and intersect only the top boundary edge of $C$. This completes the proof.

\subsection{Las-Vegas-Type Data Structures}

The lower bounds we have proved so far apply to data structures with deterministic construction algorithms and worst-case query bounds. In many cases, however, it is significantly easier to obtain efficient randomized data structures of the Las Vegas kind, that is, with expected query bounds resulting from randomness in their construction and in the query procedure (see, e.g., [17]). Therefore, we obtain a much stronger statement of the difficulty of cache-oblivious range reporting if we can show that the lower bounds shown in the previous sections apply also to this type of data structure.

Formally, we consider data structures whose construction algorithm and query procedure both have access to a sequence of random bits. The random bits used during the construction influence the shape of the data structure, while the random bits used by the query procedure influence which blocks are read to answer a given query. In a Las-Vegas-type data structure, the random bits may influence the costs of individual queries, but the answer provided by a query must always be correct. The following theorem extends the lower bounds we have proved for deterministic data structures with worst-case query bounds to randomized data structures of the Las Vegas kind.

Theorem 6 Let $f(\cdot, \cdot)$ be a monotonically increasing function, and $0<\delta \leq 1 / 2$ a constant. Any cache-oblivious data structure for three-sided range reporting, 3-d dominance reporting or 3-d halfspace range reporting constructed by a randomized algorithm and capable of answering queries using at most $f\left(\log _{B} N, K / B\right)$ block 
transfers in the expected sense, for every block size $B \leq N^{2 \delta}$, must use expected $\Omega\left(N(\log \log N)^{\varepsilon}\right)$ space, where $\varepsilon=1 /\left(4 f\left(\delta^{-1}, 1\right)\right)$.

A cache-oblivious persistent B-tree that supports 1 -d range reporting queries on any previous version of the tree using at most $f\left(\log _{B} N, K / B\right)$ block transfers in the expected sense, for every block size $B \leq N^{2 \delta}$, must use expected $\Omega\left(N(\log \log N)^{\varepsilon}\right)$ space to represent a sequence of $N$ update operations.

To prove Theorem 6, it suffices to prove it for three-sided range reporting and $\delta=1 / 2$. As before, Lemma 2 then extends the result to smaller values of $\delta$, and the reductions in Sects. 5.1 and 5.2 extend the lower bound to 3-d dominance reporting, 3-d halfspace range reporting, and cache-oblivious persistent B-trees.

Again, we formulate our proof in the multi-level indexability model from Sect. 2. To do so, we need to extend the model to allow for randomness in the construction of the indexing scheme. The randomness in the query procedure can be eliminated using the following argument. A randomized query procedure for an indexing scheme $\mathcal{I}$ would make random choices in selecting the blocks to cover a given query $q$. The efficiency of an indexing scheme, however, is defined based on the existence of a small set of blocks to cover each query $q \in Q$. This is equivalent to a query procedure that deterministically selects the minimum number of blocks to cover each query in $Q$, and randomness cannot improve on this query cost.

As a model of randomness in the construction of an indexing scheme for a workload $\mathcal{W}=(S, Q, \mathcal{B})$, we define the concepts of a randomized block cover and of a randomized indexing scheme. If the construction of an indexing scheme uses $b$ random bits, it is capable of constructing $n:=2^{b}$ different indexing schemes $\mathcal{I}^{1}, \mathcal{I}^{2}, \ldots, \mathcal{I}^{n}$, depending on the value of these bits. For a block size $B_{i} \in \mathcal{B}$, let $\mathcal{C}_{i}^{k}$ be the $B_{i}$-cover in $\mathcal{I}^{k}$. Then we call the set $\mathcal{C}_{i}=\left\{\mathcal{C}_{i}^{1}, \mathcal{C}_{i}^{2}, \ldots, \mathcal{C}_{i}^{n}\right\}$ a randomized $B_{i}$-cover and the set $\mathcal{I}=\left\{\mathcal{I}^{1}, \mathcal{I}^{2}, \ldots, \mathcal{I}^{n}\right\}$ a randomized indexing scheme for $\mathcal{W}$. The expected size of $\mathcal{I}$ is the average size of $\mathcal{I}^{1}, \mathcal{I}^{2}, \ldots, \mathcal{I}^{n}$. We say the randomized $B_{i}$-cover $\mathcal{C}_{i}$ in $\mathcal{I}$ is $f$-efficient if the average number of blocks needed to cover each query in $Q$ is at most $f\left(\log _{B_{i}} N, K / B_{i}\right)$, where the average is taken over all block covers $\mathcal{C}_{i}^{1}, \mathcal{C}_{i}^{2}, \ldots, \mathcal{C}_{i}^{n}$ in $\mathcal{C}_{i}$. The randomized indexing scheme $\mathcal{I}$ is $f$-efficient if all its randomized block covers are $f$-efficient. As such, the efficiency of a randomized indexing scheme and its expected size capture the expected query cost and the expected size of a cache-oblivious data structure, assuming that each of the $n$ data structures that can be constructed using $b$ random bits is equally likely.

To prove Theorem 6 , we show now that an $f$-efficient randomized indexing scheme $\mathcal{I}=\left\{\mathcal{I}^{1}, \mathcal{I}^{2}, \ldots, \mathcal{I}^{n}\right\}$ for the workload $\mathcal{W}=(S, Q, \mathcal{B})$ from Sect. 3 has expected size $\Omega\left(N(\log \log N)^{\varepsilon}\right)$. To this end, we represent the construction of the point set $S$ using a tree $\mathcal{T}$. The root of $\mathcal{T}$ is the top-level grid $T$, and its internal nodes represent the subgrids constructed recursively. A grid $T^{\prime \prime}$ is a child of a grid $T^{\prime}$ in $\mathcal{T}$ if $T^{\prime \prime}$ is the subgrid constructed in one of the cells of $T^{\prime}$. Now we make $n$ copies $\mathcal{T}^{2}, \mathcal{T}^{2}, \ldots, \mathcal{T}^{n}$ of $\mathcal{T}$ and associate the copy $\mathcal{T}^{k}$ with the indexing scheme $\mathcal{I}^{k}$, for all $1 \leq k \leq n$. We refer to the copy of a node $T^{\prime} \in \mathcal{T}$ in $\mathcal{T}^{k}$ as $T_{k}^{\prime}$, and we say that a point $p$ in $T_{k}^{\prime}$ has multiplicity $m$ if its multiplicity in $\mathcal{I}^{k}$ is $m$.

To prove the desired lower bound on the expected size of $\mathcal{I}$, we mimic the proof of Lemma 1 . To prove Lemma 1 , we first showed that every grid $T^{\prime} \in \mathcal{T}$ has a cell 
at least half of whose points have multiplicity $\Omega\left(t^{\varepsilon}\right)$ in an $f$-efficient (deterministic) indexing scheme $\mathcal{I}$. By Lemma 3, this implied Lemma 1. This proof was based on the fact that every query $q \in Q_{T^{\prime}}$ can be covered with at most $\alpha$ blocks in the $B_{T^{\prime}}$-cover in $\mathcal{I}$. In a randomized indexing scheme $\left\{\mathcal{I}^{1}, \mathcal{I}^{2}, \ldots, \mathcal{I}^{n}\right\}$, there may be no indexing scheme $\mathcal{I}^{k}$ whose $B_{T^{\prime}}$-cover can cover every query in $Q_{T^{\prime}}$ with at most $\alpha$ blocks. Nevertheless, we can prove that at least $n / 4$ of the copies $T_{1}^{\prime}, T_{2}^{\prime}, \ldots, T_{n}^{\prime}$ of each grid $T^{\prime} \in \mathcal{T}$ have a cell at least half of whose points have multiplicity $\Omega\left(t^{\varepsilon}\right)$ (Lemma 7 below). This suffices to show that the total size of the indexing schemes $\mathcal{I}^{1}, \mathcal{I}^{2}, \ldots, \mathcal{I}^{n}$ is $\Omega\left(n N(\log \log N)^{\varepsilon}\right)$, that is, their average size is $\Omega\left(N(\log \log N)^{\varepsilon}\right)$.

Lemma 7 Every node $T^{\prime} \in \mathcal{T}$ has at least $n / 4$ copies among $T_{1}^{\prime}, T_{2}^{\prime}, \ldots, T_{n}^{\prime}$ such that each has a cell at least half of whose points have multiplicity $\Omega\left(t^{\varepsilon}\right)$.

Proof Consider the $B_{i}$-covers $\mathcal{C}_{i}^{1}, \mathcal{C}_{i}^{2}, \ldots, \mathcal{C}_{i}^{n}$, where $B_{i}=B_{T^{\prime}}$ is the block size in $\mathcal{B}$ corresponding to the grid $T^{\prime}$. We consider a query $q \in Q_{T^{\prime}}$ cheap for $\mathcal{C}_{i}^{k}$ if $q$ can be covered using at most $4 f(2,1)$ blocks in $\mathcal{C}_{i}^{k}$, and expensive otherwise. Since the randomized $B_{i}$-cover $\mathcal{C}_{i}$ is $f$-efficient, $q$ must be cheap for at least $3 n / 4$ of the $B_{i}$-covers $\mathcal{C}_{i}^{1}, \mathcal{C}_{i}^{2}, \ldots, \mathcal{C}_{i}^{n}$. The $j$ th row of $T_{k}^{\prime}$ is cheap if at least half of the level- $j$ queries in $Q_{T^{\prime}}$ are cheap for $\mathcal{C}_{i}^{k}$; otherwise the row is expensive. As each query is cheap for at least $3 n / 4$ of the $B_{i}$-covers $\mathcal{C}_{i}^{1}, \mathcal{C}_{i}^{2}, \ldots, \mathcal{C}_{i}^{n}$, every row of $T^{\prime}$ is cheap in at least $n / 2$ of the copies $T_{1}^{\prime}, T_{2}^{\prime}, \ldots, T_{n}^{\prime}$. Thus, the total number of cheap rows over all copies of $T^{\prime}$ is at least $t n / 2$, which implies that there are at least $n / 4$ copies of $T^{\prime}$ that have at least $t / 3$ cheap rows each. We show that each such copy $T_{k}^{\prime}$ has a cell at least half of whose points have multiplicity $\Omega\left(t^{\varepsilon}\right)$.

The block cover $\mathcal{C}_{i}^{k}$ defines a colouring of the points in $T_{k}^{\prime}$ and of the queries in $Q_{T^{\prime}}$ as in Sect. 3.2. For a subset $Z$ of cells of $T_{k}^{\prime}$, we call a point $p$ in a cell $c$ in row $j$ of $T_{k}^{\prime}$ weakly $Z$-exposed if no cheap query $q \in Q_{Z}$ that belongs to a level less than $j$ in $Q_{T_{k}^{\prime}}$ covers $p$, that is, if it is $Z$-exposed in the sense defined in Sect. 3.2. Point $p$ is strongly $Z$-exposed if it is weakly $Z$-exposed and the query $q_{c}$ is cheap for $\mathcal{C}_{i}^{k}$. As in Sect. 3.2, we call a cell of $T_{k}^{\prime}$ weakly or strongly $Z$-exposed if at least half its points are weakly or strongly $Z$-exposed. Now it suffices to show that $T_{k}^{\prime}$ contains a set $Z:=\left\{c_{1}, c_{2}, \ldots, c_{s}\right\}$ of cells in the same column whose length is $s=\Omega(t / \log \alpha)$ and all of whose cells are strongly $Z$-exposed, for $\alpha:=4 f(2,1)$. Since each query $q_{c_{i}}$ is $\alpha$-coloured in this case, Lemma 5 then shows that the $Z$-exposed points in at least one of these cells have at least $s^{\varepsilon}=\Omega\left(t^{\varepsilon}\right)$ colours. This finishes the proof because at least half the points in a $Z$-exposed cell are $Z$-exposed.

Let $T^{c}$ be the subgrid of $T_{k}^{\prime}$ consisting of only the cheap rows of $T_{k}^{\prime}$, and, similarly to the proof of Lemma 4 , let $T_{h}^{c}$ be the subgrid of $T^{c}$ consisting of rows $1, h+1$, $2 h+1, \ldots$ of $T^{c}$. Using the same arguments as in the proof of Lemma 4, at most a $\left(4 \alpha / 2^{h}\right)$-fraction of the points in each row of $T_{h}^{c}$ can be covered by cheap queries at higher levels of $T_{h}^{c}$. Thus, for $h=\lceil\log (32 \alpha)\rceil$, at least a $7 / 8$-fraction of the points in each row of $T_{h}^{c}$ are weakly $T_{h}^{c}$-exposed. Since a cell is weakly $T_{h}^{c}$-exposed if at least half of its points are weakly $T_{h}^{c}$-exposed, this implies that at least a 3/4-fraction of the cells in each row of $T_{h}^{c}$ are weakly $T_{h}^{c}$-exposed. It follows that at least a $1 / 4-$ fraction of the cells in each row are strongly $T_{h}^{c}$-exposed because every row of $T_{h}^{c}$ is cheap. This, however, implies that there exists a column of $T_{h}^{c}$ such that at least 
a 1/4-fraction of its cells are strongly $T_{h}^{c}$-exposed. Since the height of $T_{h}^{c}$ is at least $t /(3 h)=\Omega(t / \log \alpha)$, this shows that $T_{k}^{\prime}$ contains a set $Z$ of $\Omega(t / \log \alpha)$ strongly $T_{h}^{c}$-exposed cells in the same column. These cells are also strongly $Z$-exposed because $Z \subseteq T_{h}^{c}$.

Using Lemma 7, we can now prove that the average size of the indexing schemes $\mathcal{I}^{1}, \mathcal{I}^{2}, \ldots, \mathcal{I}^{n}$ - that is, the expected size of $\mathcal{I}$-is $\Omega\left(N(\log \log N)^{\varepsilon}\right)$. To this end, we call a node $T_{k}^{\prime} \in \mathcal{T}^{k}$ accounted for if there exists an ancestor $T_{k}^{\prime \prime}$ of $T_{k}^{\prime}$ in $\mathcal{T}^{k}$ for which we can guarantee that at least half the points in $T_{k}^{\prime \prime}$ have multiplicity $\Omega\left(t^{\varepsilon}\right)$. We call a point accounted for if it is contained in a grid $T_{k}^{\prime}$ that is accounted for. Our goal now is to show that there exists a level $i$ in $\mathcal{T}$ such that at least a constant fraction of the points in all level- $i$ nodes of trees $\mathcal{T}^{1}, \mathcal{T}^{2}, \ldots, \mathcal{T}^{n}$ are accounted for. Since at least half of the accounted-for points have multiplicity $\Omega\left(t^{\varepsilon}\right)$, this shows that the total size of the indexing schemes $\mathcal{I}^{1}, \mathcal{I}^{2}, \ldots, \mathcal{I}^{n}$ is $\Omega\left(n N t^{\varepsilon}\right)$, and their average size is $\Omega\left(N t^{\varepsilon}\right)=\Omega\left(N(\log \log N)^{\varepsilon}\right)$, as claimed.

For a given level $i$ in $\mathcal{T}$, we consider all nodes at level $i$ in $\mathcal{T}$ that have more than $7 n / 8$ unaccounted-for copies in trees $\mathcal{T}^{1}, \mathcal{T}^{2}, \ldots, \mathcal{T}^{n}$. Let $N_{i}$ be the total number of points in these nodes of $\mathcal{T}$. By Lemma 7, each such node $T^{\prime}$ has at least $n / 8$ copies in trees $\mathcal{T}^{1}, \mathcal{T}^{2}, \ldots, \mathcal{T}^{n}$ that are unaccounted for and such that each has a child that is accounted for at level $i+1$. If $T^{\prime}$ contains $N^{\prime}$ points, then any child of $T^{\prime}$ contains at least $N^{\prime} / 4^{t}$ points, as argued in Sect. 3.1. Hence, at least $(n / 8) \cdot\left(N_{i} / 4^{t}\right)$ new points are accounted for at level $i+1$.

For $N_{i}>N / 2$, this shows that at least $n N /\left(16 \cdot 4^{t}\right)=n N /(16 \sqrt{\log N})$ new points are accounted for at depth $i+1$. Since there are only $n N$ points in total, this implies that there can be at most $16 \sqrt{\log N}$ levels in $\mathcal{T}$ that satisfy $N_{i}>N / 2$. However, we have shown in Sect. 3.1 that the height of $\mathcal{T}$ is at least $\log N / \log \log N$, which is greater than $16 \sqrt{\log N}$, for $N$ sufficiently large. Hence, there exists a level $i$ in $\mathcal{T}$ with $N_{i} \leq N / 2$, and at least $N / 2$ of the points in $S$ are accounted for in at least $n / 8$ of the trees $\mathcal{T}^{1}, \mathcal{T}^{2}, \ldots, \mathcal{T}^{n}$. Since at least half of the accounted for points have multiplicity $\Omega\left(t^{\varepsilon}\right)$, the total size of the indexing schemes $\mathcal{I}^{1}, \mathcal{I}^{2}, \ldots, \mathcal{I}^{n}$ is thus $\Omega\left(n N t^{\varepsilon}\right)$, as claimed. This concludes the proof of Theorem 6 .

\section{Conclusions}

In this paper, we have provided another separation result between the cache-oblivious model and the I/O model by proving an $\Omega\left((\log \log N)^{\varepsilon}\right)$ gap between the space bounds of range reporting data structures with optimal query bounds in the two models. While previous separation results between the two models had been obtained (with considerable technical difficulty and using sophisticated techniques), our result is the first one that concerns the size of data structures in the two models.

Our lower bound still leaves a sizable gap to the $\mathrm{O}(N \log N)$ space bound required by the currently best cache-oblivious data structures for the problems we considered. As our analysis in Sect. 4 shows, the result we obtained is in fact the best possible with the point and query sets we considered. Thus, it remains open whether stronger lower bounds can be obtained using harder point sets or whether the $\mathrm{O}(N \log N)$ space 
bound of the currently best data structures for these problems can be lowered. In particular, it seems plausible that $\mathrm{O}\left(N \log ^{\varepsilon} N\right)$-space data structures for these problems may exist.

\section{References}

1. Afshani, P.: On dominance reporting in 3D. In: Proceedings of the 16th European Symposium on Algorithms. Lecture Notes in Computer Science, vol. 5193, pp. 41-51. Springer, Berlin (2008). http://www.springerlink.com/content/r853v710340481u3

2. Afshani, P., Chan, T.M.: Optimal halfspace range reporting in three dimensions. In: Proceedings of the 20th ACM-SIAM Symposium on Discrete Algorithms, pp. 180-186 (2009)

3. Afshani, P., Hamilton, C., Zeh, N.: A general approach for cache-oblivious range reporting and approximate range counting. In: Proceedings of the 25th ACM Symposium on Computational Geometry, pp. 287-295 (2009)

4. Agarwal, P.K., Arge, L., Erickson, J., Franciosa, P.G., Vitter, J.S.: Efficient searching with linear constraints. J. Comput. Syst. Sci. 61, 194-216 (2000)

5. Agarwal, P.K., Arge, L., Danner, A., Holland-Minkley, B.: Cache-oblivious data structures for orthogonal range searching. In: Proceedings of the 19th ACM Symposium on Computational Geometry, pp. 237-245 (2003)

6. Aggarwal, A., Vitter, J.S.: The input/output complexity of sorting and related problems. Commun. ACM 31(9), 1116-1127 (1988)

7. Arge, L., Zeh, N.: Simple and semi-dynamic structures for cache-oblivious orthogonal range searching. In: Proceedings of the 22nd ACM Symposium on Computational Geometry, pp. 158-166 (2006)

8. Arge, L., Samoladas, V., Vitter, J.S.: On two-dimensional indexability and optimal range search indexing. In: Proceedings of the 18th Symposium on Principles of Database Systems, pp. 346-357 (1999)

9. Arge, L., de Berg, M., Haverkort, H.J., Yi, K.: The priority R-tree: a practically efficient and worstcase optimal R-tree. In: Proceedings of the ACM SIGMOD International Conference on Management of Data, pp. 347-358 (2004)

10. Arge, L., Brodal, G.S., Fagerberg, R., Laustsen, M.: Cache-oblivious planar orthogonal range searching and counting. In: Proceedings of the 21st ACM Symposium on Computational Geometry, pp. 160169 (2005)

11. Arge, L., de Berg, M., Haverkort, H.J.: Cache-oblivious R-trees. In: Proceedings of the 21st ACM Symposium on Computational Geometry, pp. 170-179 (2005)

12. Bayer, R., McCreight, E.M.: Organization and maintenance of large ordered indices. Acta Inform. 1, 173-189 (1972)

13. Becker, B., Gschwind, S., Ohler, T., Seeger, B., Widmayer, P.: An asymptotically optimal multiversion B-tree. VLDB J. 5(4), 264-275 (1996)

14. Bender, M.A., Brodal, G.S., Fagerberg, R., Ge, D., He, S., Hu, H., Iacono, J., López-Ortiz, A.: The cost of cache-oblivious searching. In: Proceedings of the 44th IEEE Symposium on Foundations of Computer Science, pp. 271-282 (2003)

15. Brodal, G.S., Fagerberg, R.: On the limits of cache-obliviousness. In: Proceedings of the 35th ACM Symposium on Theory of Computing, pp. 307-315 (2003)

16. Chan, T.M.: Random sampling, halfspace range reporting, and construction of $(\leq k)$-levels in three dimensions. In: Proceedings of the 39th IEEE Symposium on Foundations of Computer Science, pp. 586-595 (1998)

17. Chan, T.M.: Random sampling halfspace range reporting, and construction of $(\leq k)$-levels in three dimensions. SIAM J. Comput. 30(2), 561-575 (2000)

18. Frigo, M., Leiserson, C.E., Prokop, H., Ramachandran, S.: Cache-oblivious algorithms. In: Proceedings of the 40th IEEE Symposium on Foundations of Computer Science, pp. 285-397 (1999)

19. Grossi, R., Italiano, G.F.: Efficient cross-tree for external memory. In: Abello, J., Vitter, J.S. (eds.) External Memory Algorithms and Visualization, pp. 87-106. American Mathematical Society, New York (1999)

20. Grossi, R., Italiano, G.F.: Efficient splitting and merging algorithms for order decomposable problems. Inf. Comput. 154(1), 1-33 (1999) 
21. Hellerstein, J.M., Koutsoupias, E., Papadimitriou, C.H.: On the analysis of indexing schemes. In: Proceedings of the 16th ACM Symposium on Principles of Database Systems, pp. 249-256 (1997)

22. Kanth, K.V.R., Singh, A.K.: Optimal dynamic range searching in non-replicated index structures. In: Proceedings of the International Conference on Database Theory. Lecture Notes in Computer Science, vol. 1540, pp. 257-276. Springer, Berlin (1999)

23. Koutsoupias, E., Taylor, D.S.: Tight boundns for 2-dimensional indexing schemes. In: Proceedings of the 17th ACM Symposium on Principles of Database Systems, pp. 52-58 (1998)

24. Makris, C., Tsakalidis, A.: Algorithms for three-dimensional dominance searching in linear space. Inf. Process. Lett. 66(6), 277-283 (1998). doi:10.1016/S0020-0190(98)00075-1

25. McCreight, E.M.: Priority search trees. SIAM J. Comput. 14(2), 257-276 (1985)

26. Procopiuc, O., Agarwal, P.K., Arge, L., Vitter, J.S.: Bkd-tree: a dynamic scalable kd-tree. In: Proceedings of the 8th International Symposium on Advances in Spatial and Temporal Databases. Lecture Notes in Computer Science, vol. 2750, pp. 46-65. Springer, Berlin (2003)

27. Ramos, E.A.: On range reporting, ray shooting and $k$-level construction. In: Proceedings of the 15 th ACM Symposium on Computational Geometry, pp. 390-399 (1999)

28. Robinson, J.: The K-D-B tree: a search structure for large dimensional dynamic indexes. In: Proceedings of the SIGMOD International Conference on Management of Data, pp. 10-18 (1981)

29. Samoladas, V., Miranker, D.P.: A lower bound theorem for indexing schemes and its application to multidimensional range queries. In: Proceedings of the 17th ACM Symposium on Principles of Database Systems, pp. 44-51 (1998)

30. Vengroff, D.E., Vitter, J.S.: Efficient 3-D range searching in external memory. In: Proceedings of the 28th ACM Symposium on Theory of Computing, pp. 192-201 (1996)

31. Vitter, J.S.: External memory algorithms and data structures: dealing with massive data. ACM Comput. Surv. 33(2) (2001). Updated version at http://www.cs.purdue.edu/ jsv/Papers/Vit.IO_survey.pdf 